\title{
Synthesis, Structural Characterization, and Biological Activity Studies of Ni(II) and Zn(II) Complexes
}

\author{
Palakuri Kavitha and K. Laxma Reddy \\ Department of Chemistry, National Institute of Technology, Warangal 506 004, India \\ Correspondence should be addressed to K. Laxma Reddy; laxmareddychem12@gmail.com
}

Received 28 October 2013; Accepted 26 March 2014; Published 28 April 2014

Academic Editor: Imre Sovago

Copyright (C) 2014 P. Kavitha and K. L. Reddy. This is an open access article distributed under the Creative Commons Attribution License, which permits unrestricted use, distribution, and reproduction in any medium, provided the original work is properly cited.

$\mathrm{Ni}(\mathrm{II})$ and $\mathrm{Zn}$ (II) complexes were synthesized from tridentate 3-formyl chromone Schiff bases such as 3-((2-hydroxyphenylimino)methyl)-4H-chromen-4-one $\left(\mathrm{HL}_{1}\right), 2-\left(\left(4-\right.\right.$ oxo-4H-chromen-3-yl)methylneamino)benzoic acid $\left(\mathrm{HL}_{2}\right)$, 3-((3-hydroxypyridin-2-ylimino)methyl)-4H-chromen-4-one $\left(\mathrm{HL}_{3}\right)$, and 3-((2-mercaptophenylimino)methyl)-4H-chromen-4-one $\left(\mathrm{HL}_{4}\right)$. All the complexes were characterized in the light of elemental analysis, molar conductance, FTIR, UV-VIS, magnetic, thermal, powder $\mathrm{XRD}$, and SEM studies. The conductance and spectroscopic data suggested that, the ligands act as neutral and monobasic tridentate ligands and form octahedral complexes with general formula $\left[\mathrm{M}\left(\mathrm{L}_{1-4}\right) 2\right] \cdot n \mathrm{H}_{2} \mathrm{O}(\mathrm{M}=\mathrm{Ni}(\mathrm{II})$ and $\mathrm{Zn}(\mathrm{II}))$. Metal complexes exhibited pronounced activity against tested bacteria and fungi strains compared to the ligands. In addition metal complexes displayed good antioxidant and moderate nematicidal activities. The cytotoxicity of ligands and their metal complexes have been evaluated by MTT assay. The DNA cleavage activity of the metal complexes was performed using agarose gel electrophoresis in the presence and absence of oxidant $\mathrm{H}_{2} \mathrm{O}_{2}$. All metal complexes showed significant nuclease activity in the presence of $\mathrm{H}_{2} \mathrm{O}_{2}$.

\section{Introduction}

Metal complexes of O, S, and N containing Schiff bases have been the subject of current and growing interest because it has wide range of pharmacological activities [1]. In particular Schiff bases with 2-amino thiophenol, 2-amino phenol, 2amino benzoic acid, and 2-amino 3-hydroxy pyridine exhibit various biological activities such as antimicrobial activity, protein tyrosine phosphatases inhibition, and nuclease activity $[2,3]$. Several aromatic amine Schiff bases have been investigated but few works deal with chromone skeleton derivatives. Chromones are a group of naturally occurring compounds that are ubiquitous in nature especially in plants. Molecules containing the chromone skeleton have extensive biological applications including antimycobacterial, antifungal, anticancer, antioxidant, antihypertensive, and antiinflammatory applications and tyrosinase and protein kinase C inhibitors [4-13].

DNA plays an important role in the life process since it contains all the genetic information for the cellular function. However, DNA is the primary intracellular target of anticancer drugs, damaged under various conditions such as interactions with some small molecules, which cause DNA damage in cancer cells blocking the division of cancer cells and resulting in cell death. Metal complexes such as cobalt, nickel, copper, and zinc with Schiff base ligands have shown excellent binding and cleavage activities [14-16]. There is substantial literature supporting the DNA binding studies of chromone Schiff bases and their metal complexes [17-19]. Compounds showing the properties of effective binding as well as cleaving double stranded DNA under physiological conditions are of great importance since these could be used as diagnostic agents in medicinal and genomic research.

Fluorescent transition metal centres are particularly attractive moieties because they often possess distinctive electrochemical or photophysical properties, thus enhancing the functionality of the binding agent [20].

In previous papers we presented synthesis, detailed characterization, and biological activity studies of 3-formyl chromone Schiff bases such as 3-((2-hydroxy phenylimino)methyl)-4H-chromen-4-one $\left(\mathrm{HL}_{1}\right), 2-((4-0 x 0-4 \mathrm{H}$-chromen3-yl)methylneamino)benzoic acid $\left(\mathrm{HL}_{2}\right), 3$-((3-hydroxypy- 
ridin-2-ylimino)methyl)-4H-chromen-4-one $\left(\mathrm{HL}_{3}\right)$, 3-((2mercapto phenylimino)methyl)-4H-chromen-4-one $\left(\mathrm{HL}_{4}\right)$, and their $\mathrm{Cu}(\mathrm{II}), \mathrm{Co}(\mathrm{II})$, and $\mathrm{Pd}(\mathrm{II})$ complexes [21-23]. The present paper deals with the synthesis, characterization of $\mathrm{Ni}$ (II) and $\mathrm{Zn}$ (II) complexes of 3-formyl chromone Schiff bases $\left(\mathrm{HL}_{1}, \mathrm{HL}_{2}, \mathrm{HL}_{3}\right.$, and $\left.\mathrm{HL}_{4}\right)$, and various biological activity studies, that is, antimicrobial, antioxidant, and nematicidal activities and cytotoxicity and DNA cleavage.

\section{Experimental}

2.1. Materials and Physical Measurements. All the chemicals used were of analytical grade and procured from Spectrochem Pvt. Ltd., Mumbai, India. 3-Formyl chromone was synthesized according to the literature [24].

The elemental analysis of carbon, hydrogen, nitrogen, and sulphur contents was performed using Perkin Elmer CHNS analyser. Molar conductance of the complexes was measured using a Digisun conductivity meter in DMF. The electronic absorption spectra of the complexes were recorded on JASCO V-670 Spectrophotometer in the wavelength region of 250$1400 \mathrm{~nm}$ in the solid state. The FTIR spectra of the complexes were recorded on Tensor 2 FTIR spectrophotometer in the region of $4000-400 \mathrm{~cm}^{-1}$ using $\mathrm{KBr}$ disc. The magnetic susceptibilities of $\mathrm{Ni}$ (II) complexes were measured with a Sherwood scientific balance. Diamagnetic corrections were calculated from Pascal's constants. The magnetic moment values were calculated using the relation $\mu_{\text {eff }}=2.83\left(\chi_{m} T\right)^{1 / 2}$ B.M. Thermal studies of the complexes were carried out on a Perkin Elmer diamond TGA instrument at a heating rate of $10^{\circ} \mathrm{C}$ and nitrogen flow rate of $20 \mathrm{~mL} / \mathrm{min}$. The fluorescence spectra of the complexes were recorded on Fluorolog FL3-11 spectrofluorometer. The X-ray patterns of the complexes were recorded on Xpert-Pro X-ray diffractometer with $\mathrm{Cu} K \alpha$ radiation $(\lambda=$ $1.5406 \AA$ ). The diffraction data are integrated by using the Nakamuta program. Scanning electron micrograph (SEM) of the complexes was obtained in a Hitachi S-520 electron microscope at an accelerated voltage of $15 \mathrm{kV}$.

2.2. Synthesis of Ligands. All the Schiff base ligands $\mathrm{HL}_{1}$, $\mathrm{HL}_{2}, \mathrm{HL}_{3}$, and $\mathrm{HL}_{4}$ (Figure 1) were prepared according to the literature methods $[21,25,26]$.

2.3. Synthesis of Metal Complexes. The ligands (2 mM) and metal acetates $\left(\mathrm{Ni}\left(\mathrm{CH}_{3} \mathrm{COOH}\right)_{2} \cdot 4 \mathrm{H}_{2} \mathrm{O}\right.$ and $\mathrm{Zn}\left(\mathrm{CH}_{3}\right.$ $\left.\mathrm{COOH})_{2} \cdot 2 \mathrm{H}_{2} \mathrm{O}\right)(1 \mathrm{mM})$ were dissolved in methanol. The mixtures were stirred at room temperature for 2-3 h; various colour precipitates were separated from the solution by suction filtration, purified by washing several times with methanol, and dried for $12 \mathrm{~h}$ in vacuum.

2.4. Antimicrobial Activity. In vitro antimicrobial activity of the metal complexes towards the bacteria Proteus vulgaris, Klebsiella pnuemoniae, Staphylococcus aureus, and Bacillus subtili and fungi Candida albicans was carried out using disc diffusion method. The antibiotics kanamycin and clotrimazole are the standards for antibacterial and antifungal activity studies. Standard inoculum, 1-2 × $10^{7} \mathrm{cfu} / \mathrm{mL} 0.5 \mathrm{Mc}$ Farland

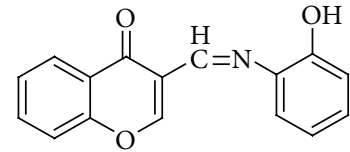

3-(-(2-Hydroxyphenylimino)methyl)-4H-chromen-4-one $\left(\mathrm{HL}_{1}\right)$

(a)<smiles>O=C(O)c1ccccc1N=Cc1coc2ccccc2c1=O</smiles>

2-((4-Oxo-4H-chromen-3-yl)methyleneamino)benzoic acid $\left(\mathrm{HL}_{2}\right)$

(b)<smiles>O=c1c(/C=N/c2ncccc2O)coc2ccccc12</smiles>

3-((3-Hydroxypyridin-2-ylimino)methyl)-4H-chromen-4-one $\left(\mathrm{HL}_{3}\right)$

(c)<smiles>O=c1c(/C=N/c2ccccc2S)coc2ccccc12</smiles>

3-(-(2-Mercaptophenylimino)methyl)-4H-chromen-4-one $\left(\mathrm{HL}_{4}\right)$

(d)

Figure 1: Structures of Schiff base ligands.

standards [27], was introduced onto the surface of sterile nutrient agar plate and evenly spread by using a sterile glass spreader. Sterile antibiotic discs $(6 \mathrm{~mm}$ in diameter, prepared using Whatmann number 1 paper) were placed over the nutrient agar medium. Each disc was spread by $100 \mu \mathrm{g}$ of the compounds (initially dissolved in DMSO). The plates were incubated with bacterial cultures for $24 \mathrm{~h}$ at $37^{\circ} \mathrm{C}$ and fungal cultures at $25^{\circ} \mathrm{C}$ for $48 \mathrm{~h}$. The activity of the compounds was determined by measuring the diameter of inhibition zone in "millimetres" and compared with standard antibiotics. DMSO (which has no activity) and standard antibiotics were used as negative and positive controls for antimicrobial activity studies. The activity results are calculated as a mean of triplicates.

Minimum inhibitory concentrations (MIC) of the complexes which showed antimicrobial activity were determined using the literature method [28]. All the compounds that were diluted within the range of $100-10 \mu \mathrm{g} / \mathrm{mL}$ were mixed in nutrient broth and $0.1 \mathrm{~mL}$ of active inoculums was added to each tube. The tubes were incubated aerobically at $37^{\circ} \mathrm{C}$ for bacteria and $25^{\circ} \mathrm{C}$ for fungi up to $24 \mathrm{~h}$. The lowest concentration of the compound that completely inhibited 
bacterial growth (no turbidity) in comparison to control was regarded as MIC.

2.5. Nematicidal Activity. Root knot nematode, Meloidogyne incognita, is major plant parasitic nematodes affecting quantity and quality of the crop production in many annual and perennial crops. Meloidogyne nematode can develop galls and lesions in the roots, thereby causing stunted growth of the plants. Some of the chemicals can be used to control nematodes [29].

Nematicidal activity of the complexes was carried out on Meloidogyne incognita. Fresh egg masses of Meloidogyne incognita are collected from stock culture maintained on tomato (Lycopersicon esculentum) root tissues and kept in water for egg hatching. The eggs suspensions were poured on a cotton wool filter paper and incubated at $30^{\circ} \mathrm{C}$ to obtain freshly hatched juveniles (J2). Juveniles collected within $48 \mathrm{~h}$ were used for screening nematicidal activity of the compounds.

The compounds were initially dissolved in dimethyl sulfoxide (DMSO) and then in distilled water to make dilutions of 250,150 , and $50 \mu \mathrm{g} / \mathrm{mL}$. Experiments were performed under laboratory conditions at $30^{\circ} \mathrm{C}$. About 100 freshly hatched second stage juveniles were suspended in $5 \mathrm{~mL}$ of each diluted compound and incubated. Distilled water with nematode larvae was taken as control. The dead nematodes were observed under an inverted binocular microscope. After an incubation of 24 and $48 \mathrm{~h}$, percentage of mortality was calculated. Nematodes were considered dead if they did not move when probed with a fine needle [30].

2.6. DPPH Radical Scavenging Activity. The free radical scavenging activities of the metal complexes were determined by using DPPH free radical scavenging method according to the literature [31]. DPPH is a stable free radical containing an odd electron in its structure and usually utilized for detection of the radical scavenging activity in chemical analysis. In the spectrophotometric assay the ability to scavenge the stable free radical DPPH is measured by decrease in the absorbance at $517 \mathrm{~nm}$. Each compound was dissolved in methanol $(10 \mathrm{mg} / 10 \mathrm{~mL})$ and it was used as stock solution. From the stock solutions, $1 \mathrm{~mL}$ of each compound solution with different concentrations $(0.25 \mu \mathrm{g}-1.00 \mu \mathrm{g})$ was added to the $3 \mathrm{~mL}$ of methanolic DPPH $(0.004 \%)$ solution. After $30 \mathrm{~min}$, the absorbance of the test compounds was taken at $517 \mathrm{~nm}$ using UV-VIS spectrophotometer. BHT was used as standard, DPPH solution was used as control without the test compounds, and methanol was used as blank. The percentage of scavenging activity of DPPH free radical was measured by using the following formula:

$$
\text { Scavenging activity }(\%)=\left[\frac{\left(A_{o}-A_{i}\right)}{A_{o}}\right] \times 100,
$$

where $A_{o}$ is the absorbance of the control and $A_{i}$ is the absorbance of the sample.

2.7. Cytotoxic Activity. The human breast carcinoma cell line (MCF-7), human colon carcinoma cell line (COLO
205), and murine microphage cell line (Raw 264.7) were obtained from the National Centre for Cell Science (NCCS), Pune, and grown in Dulbecco's Modified Eagles Medium (DMEM) containing 10\% fetal bovine serum (FBS), amphotericin $(3 \mu \mathrm{g} / \mathrm{mL})$, gentamycin $(400 \mu \mathrm{g} / \mathrm{mL})$, streptomycin $(250 \mu \mathrm{g} / \mathrm{mL})$, and penicillin $(250$ units $/ \mathrm{mL})$ in a carbon dioxide incubator at $5 \% \mathrm{CO}_{2}$. About 700 cells/well were seeded in 96-well plate using culture medium; the viability was tested using trypan blue dye with help of haemocytometer and $95 \%$ of viability was confirmed. After $24 \mathrm{~h}$, the new medium with compounds in the concentration of 100,10 , and $1 \mu \mathrm{g} / \mathrm{mL}$ were added at respective wells and kept in incubation for $48 \mathrm{~h}$. After incubation MTT assay was performed.

2.7.1. MTT Assay. After $48 \mathrm{~h}$ of the drug treatment the medium was changed again for all groups and $10 \mu \mathrm{L}$ of MTT ( $5 \mathrm{mg} / \mathrm{mL}$ stock solution) was added and the plates were incubated for an additional $4 \mathrm{~h}$. The medium was discarded and the formazan blue, which was formed in the cells, was dissolved with $50 \mu \mathrm{L}$ of DMSO. The optical density was measured on microplate spectrophotometer at a wavelength of $570 \mathrm{~nm}$. The percentage of cell inhibition was calculated by using the following formula [32]:

$$
\% \text { Growth inhibition }=100-\left(\frac{A_{i}}{A_{o}}\right) \times 100 \text {, }
$$

where $A_{i}$ is the absorbance of the sample and $A_{o}$ is the absorbance of the control. IC $_{50}$ values were determined using Graph Pad Prism software.

2.8. DNA Cleavage Activity. The DNA cleavage activity of metal complexes was monitored by agarose gel electrophoresis. pUC19 plasmid was cultured, isolated, and used as DNA for the experiment. Test samples $(1 \mathrm{mg} / \mathrm{mL})$ were prepared in DMF. $25 \mu \mathrm{g}$ of the test samples was added to the isolated plasmid and incubated for $2 \mathrm{~h}$ at $37^{\circ} \mathrm{C}$. After incubation, $30 \mu \mathrm{L}$ of plasmid DNA sample mixed with bromophenol blue dye $(1: 1)$ was loaded into the electrophoresis chamber wells along with the control DNA, $5 \mathrm{M} \mathrm{FeSO}_{4}$ (treated with DNA), and standard DNA marker containing TAE buffer $(4.84 \mathrm{~g}$ Tris base, $\mathrm{pH}$ 8.0, 0.5 M EDTA/1L). Finally, it was loaded on to an agarose gel and electrophoresed at $50 \mathrm{~V}$ constant voltage up to $30 \mathrm{~min}$. After the run, gel was removed and stained with $10.01 \mu \mathrm{g} / \mathrm{mL}$ ethidium bromide and image was taken in Versadoc (Biorad) imaging system. The results were compared with standard DNA marker. The same procedure was followed in the presence of $\mathrm{H}_{2} \mathrm{O}_{2}$ also.

\section{Results and Discussion}

All the Ni(II) complexes were colored, stable, and nonhygroscopic in nature. The complexes are insoluble in common organic solvents but soluble in DMF and DMSO. The elemental analysis showed that the complexes have 1:2 stoichiometry of the type $\left[\mathrm{M}\left(\mathrm{L}_{1-4}\right)_{2}\right] \cdot n \mathrm{H}_{2} \mathrm{O}$, where $\mathrm{L}$ stands for singly deprotonated ligands. Molar conductance of the complexes was measured in DMF. The conductance values, which are 
presented in the Table 1, indicate the nonelectrolytic nature of the complexes [33].

3.1. Determination of the Metal Content of the Complexes. Known amount $(0.150 \mathrm{~g})$ of complexes was decomposed with concentrated nitric acid. This process was repeated till the organic part of the complexes got completely lost. The excess nitric acid was expelled by evaporation with concentrated sulphuric acid. The Ni(II) and $\mathrm{Zn}$ (II) contents of the complexes were determined as per the procedure available in the literature [34].

3.2. FTIR Spectra. The FTIR spectra of the complexes are compared with those of the ligands in order to determine the coordination sites that may involve in chelation. The position and/or the intensities of bands are expected to be changed while the coordination. The most important IR bands of the metal complexes with probable assignments are given in Table 2. The Schiff base ligands showing a band around $1650-1620 \mathrm{~cm}^{-1}$ is assigned to the $(\mathrm{C}=\mathrm{O})$ group of the chromone system. Upon complexation the $v(\mathrm{C}=\mathrm{O})$ group is shifted to $20-35 \mathrm{~cm}^{-1}$ lower wavenumber region [35]. The ligands show the most characteristic $\nu(\mathrm{C}=\mathrm{N})$ bands in the region $1605-1563 \mathrm{~cm}^{-1}$. In the spectra of their corresponding metal complexes, this band appears at $25-45 \mathrm{~cm}^{-1}$ to lower wavenumber region indicating the coordination of the azomethine nitrogen atom to the metal ion [36]. A broad band appeared in $\mathrm{HL}_{1}$ and $\mathrm{HL}_{3}$ at 3241 and $3246 \mathrm{~cm}^{-1}$, respectively, and is attributed to the $v(\mathrm{O}-\mathrm{H})$ group. The absence of the band in the spectra of their corresponding metal complexes is due to the involvement of oxygen atom of $(\mathrm{OH})$ group coordination to the metal ion. In $\mathrm{HL}_{2}$ ligand, a strong band appeared at $1365 \mathrm{~cm}^{-1}$ and is due to the $\nu(\mathrm{C}-\mathrm{O})$ of carboxylic group. In its metal complexes it is shifted to 18$47 \mathrm{~cm}^{-1}$ lower wavenumber region [37]. Since SH stretching frequency band is very weak, the peak corresponding to $\mathrm{SH}$ is not clearly observed in the case of $\mathrm{HL}_{4}$ ligand [38]. Presence of band around $3400 \mathrm{~cm}^{-1}$ in all the complexes is the indication of water molecules present in the complexes. The presence of oxygen and nitrogen in the coordination sphere is further confirmed by the presence of $\nu(\mathrm{M}-\mathrm{N})$ and $\nu(\mathrm{M}-\mathrm{O})$ bands at $400-600 \mathrm{~cm}^{-1}$ region. The FTIR results show that all Schiff base ligands act as tridentate chelating ligands.

3.3. Electronic Spectra and Magnetic Moments. The electronic absorption spectra of the Schiff base metal complexes in solid state were recorded at room temperature and the band positions of the absorption maxima, band assignments, ligand field parameters, and magnetic moment values are listed in Table 3. The electronic spectra of $\left[\mathrm{Ni}\left(\mathrm{L}_{4}\right)_{2}\right] \cdot \mathrm{H}_{2} \mathrm{O}$ and $\left[\mathrm{Zn}\left(\mathrm{L}_{4}\right)_{2}\right] \cdot \mathrm{H}_{2} \mathrm{O}$ complexes are depicted in Figure 2. The electronic spectra of $\mathrm{Ni}(\mathrm{II})$ complexes displayed three absorption bands in the range 8000-9000, 14000-16000, and $20000-24000 \mathrm{~cm}^{-1}$. Thus, these bands may be assigned to the three spin allowed transitions ${ }^{3} \mathrm{~A}_{2 \mathrm{~g}}(\mathrm{~F}) \rightarrow{ }^{3} \mathrm{~T}_{2 \mathrm{~g}}(\mathrm{~F})\left(\nu_{1}\right)$, ${ }^{3} \mathrm{~A}_{2 \mathrm{~g}}(\mathrm{~F}) \rightarrow{ }^{3} \mathrm{~T}_{1 \mathrm{~g}}(\mathrm{~F})\left(v_{2}\right)$, and ${ }^{3} \mathrm{~A}_{2 \mathrm{~g}}(\mathrm{~F}) \rightarrow{ }^{3} \mathrm{~T}_{1 \mathrm{~g}}(\mathrm{P})\left(v_{3}\right)$, respectively, characteristic of octahedral geometry. The values of transition ratio $\nu_{2} / \nu_{1}$ and $\beta$ lie in the range of $1.70-1.80$ and $0.89-0.95$, respectively, providing further evidence for octahedral geometry of $\mathrm{Ni}$ (II) complexes [39]. The B values for the complexes are lower than the free ion value, thereby indicating orbital overlap and delocalisation of $d$-orbitals. The $\beta$-values obtained are less than unity suggesting the covalent character of the metal-ligand bonds. All $\mathrm{Ni}$ (II) complexes are paramagnetic and the magnetic movement values at room temperature are in the range of 2.91-3.25 B.M which is well agreed with the reported octahedral $\mathrm{Ni}(\mathrm{II})$ complexes [40]. All $\mathrm{Zn}$ (II) complexes showed two bands around 25000 and $30000 \mathrm{~cm}^{-1}$ and are attributed to the $n \rightarrow \pi^{*}$ and $\pi \rightarrow$ $\pi^{*}$ transitions, respectively. $\mathrm{Zn}$ (II) complexes that are in $\mathrm{d}^{10}$ configuration are diamagnetic and do not show any $\mathrm{d}-\mathrm{d}$ transitions.

3.4. Thermal Analysis. The thermogravimetric analysis (TGA) of the metal complexes was carried out within the temperature range from room temperature to $1000^{\circ} \mathrm{C}$. The TG-DTA graphs of $\left[\mathrm{Ni}\left(\mathrm{L}_{4}\right)_{2}\right]$ and $\left[\mathrm{Zn}\left(\mathrm{L}_{4}\right)_{2}\right] \cdot \mathrm{H}_{2} \mathrm{O}$ complexes are given in Figure 3. The TGA data and their assignments of all the metal complexes are listed in Table 4. Metal complexes decompose gradually with formation of respective metal oxides. The TG graphs of $\left[\mathrm{Ni}\left(\mathrm{L}_{2}\right)_{2}\right] \cdot \mathrm{H}_{2} \mathrm{O},\left[\mathrm{Ni}\left(\mathrm{L}_{3}\right)_{2}\right] \cdot \mathrm{H}_{2} \mathrm{O}$, $\left[\mathrm{Zn}\left(\mathrm{L}_{1}\right)_{2}\right] \cdot \mathrm{H}_{2} \mathrm{O}$, and $\left[\mathrm{Zn}\left(\mathrm{L}_{4}\right)_{2}\right] \cdot \mathrm{H}_{2} \mathrm{O}$ complexes decompose in two to three steps. The first step corresponds to the loss of lattice water molecule in the temperature range between 30 and $140^{\circ} \mathrm{C}$ with a weight loss of $2-3 \%$. In the second and third steps, the total loss of ligand molecules was observed in the temperature range between 160 and $850^{\circ} \mathrm{C}$ leaving behind metal oxide as residue. The thermal decomposition of remaining metal complexes $\left(\left[\mathrm{Ni}\left(\mathrm{L}_{1}\right)_{2}\right],\left[\mathrm{Ni}\left(\mathrm{L}_{4}\right)_{2}\right]\left[\mathrm{Zn}\left(\mathrm{L}_{2}\right)_{2}\right]\right.$ and $\left.\left[\mathrm{Zn}\left(\mathrm{L}_{3}\right)_{2}\right]\right)$ occurs in two to three steps. Upon starting heating, these metal complexes losses of the organic moieties of Schiff base ligands were observed in two to three successive steps within the temperature range of $150-850^{\circ} \mathrm{C}$. In all the complexes the final mass loss is due to the formation of metal oxides as residue.

On the basis of all spectral data (elemental analysis, FTIR, electronic spectra, and thermal analysis), the suggested structures of the complexes are shown in Figure 4.

3.5. Powder XRD and SEM Studies. Single crystals of the complexes could not be obtained because of their insolubility in most organic solvents, hence, the powder diffraction data were obtained for structural characterization. The powder XRD patterns of all metal complexes were recorded in the range $2 \theta=10-50^{\circ}$. The powder XRD pattern of $\left[\mathrm{Ni}\left(\mathrm{L}_{1}\right)_{2}\right]$ and $\left[\mathrm{Zn}\left(\mathrm{L}_{1}\right)_{2}\right] \cdot \mathrm{H}_{2} \mathrm{O}$ are presented in Figure 5. Observed and calculated powder XRD data of $\left[\mathrm{Ni}\left(\mathrm{L}_{1}\right)_{2}\right]$ and $\left[\mathrm{Zn}\left(\mathrm{L}_{1}\right)_{2}\right] \cdot \mathrm{H}_{2} \mathrm{O}$ are given in Tables 5(a) and 5(b). Unit cell parameters were found by using trial and error methods. All complexes are triclinic with different unit cell parameters. All metal complexes display sharp crystalline peaks except $\left[\mathrm{Zn}\left(\mathrm{L}_{2}\right)_{2}\right]$ and $\left[\mathrm{Zn}\left(\mathrm{L}_{4}\right)_{2}\right] \cdot \mathrm{H}_{2} \mathrm{O}$; these two complexes do not exhibit welldefined crystalline peaks due to their amorphous nature. The unit cell parameters of metal complexes are as follows: $\left[\mathrm{Ni}\left(\mathrm{L}_{1}\right)_{2}\right]: a=8.80 \AA, b=8.52 \AA, c=7.50 \AA, \alpha=99.93^{\circ}$, 
TABLE 1: Elemental analysis and physical properties of metal complexes.

\begin{tabular}{|c|c|c|c|c|c|c|c|}
\hline \multirow{2}{*}{ Molecular formula } & \multirow{2}{*}{$\begin{array}{l}\text { Colour } \\
\text { (\% yield) }\end{array}$} & \multicolumn{5}{|c|}{$\begin{array}{c}\text { \% found } \\
\text { (cald.) }\end{array}$} & \multirow{2}{*}{$\begin{array}{l}\text { Molar conductivity } \\
\left(\mathrm{Ohm}^{-1} \mathrm{~cm}^{2} \mathrm{~mol}^{-1}\right)\end{array}$} \\
\hline & & $\mathrm{C}$ & $\mathrm{H}$ & $\mathrm{N}$ & S & $\begin{array}{c}M \\
(\mathrm{Ni} / \mathrm{Zn})\end{array}$ & \\
\hline$\left[\mathrm{Ni}\left(\mathrm{L}_{1}\right)_{2}\right]$ & Brick red & 64.49 & 3.21 & 4.23 & & 9.91 & 10 \\
\hline$\left[\mathrm{Ni}\left(\mathrm{C}_{16} \mathrm{H}_{10} \mathrm{O}_{3} \mathrm{~N}\right)_{2}\right]$ & (71) & $(65.45)$ & $(3.40)$ & $(4.77)$ & & $(10.00)$ & \\
\hline$\left[\mathrm{Ni}\left(\mathrm{L}_{2}\right)_{2}\right] \cdot \mathrm{H}_{2} \mathrm{O}$ & Light green & 61.59 & 3.02 & 4.21 & & 8.79 & 12 \\
\hline$\left[\mathrm{Ni}\left(\mathrm{C}_{17} \mathrm{H}_{10} \mathrm{O}_{4} \mathrm{~N}\right)_{2}\right] \cdot \mathrm{H}_{2} \mathrm{O}$ & $(62)$ & $(61.75)$ & $(3.32)$ & $(4.24)$ & & $(8.88)$ & 12 \\
\hline$\left[\mathrm{Ni}\left(\mathrm{L}_{3}\right)_{2}\right] \cdot \mathrm{H}_{2} \mathrm{O}$ & Brick red & 61.07 & 3.23 & 9.23 & & 9.43 & 15 \\
\hline$\left[\mathrm{Ni}\left(\mathrm{C}_{15} \mathrm{H}_{9} \mathrm{O}_{3} \mathrm{~N}_{2}\right)_{2}\right] \cdot \mathrm{H}_{2} \mathrm{O}$ & $(85)$ & $(61.15)$ & $(3.39)$ & $(9.51)$ & & $(9.97)$ & 10 \\
\hline$\left[\mathrm{Ni}\left(\mathrm{L}_{4}\right)_{2}\right]$ & Black & 62.21 & 3.09 & 4.61 & 9.30 & 9.21 & 14 \\
\hline$\left[\mathrm{Ni}\left(\mathrm{C}_{16} \mathrm{H}_{10} \mathrm{O}_{2} \mathrm{NS}\right)_{2}\right]$ & $(82)$ & $(62.06)$ & $(3.23)$ & $(4.52)$ & $(10.34)$ & $(9.49)$ & \\
\hline$\left[\mathrm{Zn}\left(\mathrm{L}_{1}\right)_{2}\right] \cdot \mathrm{H}_{2} \mathrm{O}$ & Orange & 62.91 & 3.52 & 4.62 & & 10.58 & 15 \\
\hline$\left[\mathrm{Zn}\left(\mathrm{C}_{16} \mathrm{H}_{10} \mathrm{O}_{3} \mathrm{~N}\right)_{2}\right] \cdot \mathrm{H}_{2} \mathrm{O}$ & (88) & $(62.80)$ & $(3.60)$ & $(4.58)$ & & $(10.70)$ & \\
\hline$\left[\mathrm{Zn}\left(\mathrm{L}_{2}\right)_{2}\right]$ & Yellow & 62.79 & 3.15 & 4.25 & & 9.95 & 11 \\
\hline$\left[\mathrm{Zn}\left(\mathrm{C}_{17} \mathrm{H}_{10} \mathrm{O}_{4} \mathrm{~N}\right)_{2}\right]$ & $(61)$ & $(62.83)$ & $(3.08)$ & $(4.31)$ & & $(10.07)$ & 11 \\
\hline$\left[\mathrm{Zn}\left(\mathrm{L}_{3}\right)_{2}\right]$ & Brick red & 60.21 & 3.25 & 9.35 & & 10.51 & 18 \\
\hline$\left[\mathrm{Zn}\left(\mathrm{C}_{15} \mathrm{H}_{9} \mathrm{O}_{3} \mathrm{~N}_{2}\right)_{2}\right]$ & (87) & $(60.46)$ & $(3.02)$ & $(9.40)$ & & $(10.98)$ & \\
\hline$\left[\mathrm{Zn}\left(\mathrm{L}_{4}\right)_{2}\right] \cdot \mathrm{H}_{2} \mathrm{O}$ & Yellow & 60.22 & 3.35 & 4.21 & 10.01 & 10.31 & 20 \\
\hline$\left[\mathrm{Zn}\left(\mathrm{C}_{16} \mathrm{H}_{10} \mathrm{O}_{2} \mathrm{NS}\right)_{2}\right] \cdot \mathrm{H}_{2} \mathrm{O}$ & $(73)$ & $(59.68)$ & $(3.41)$ & $(4.35)$ & $(9.93)$ & $(10.46)$ & \\
\hline
\end{tabular}

TABLE 2: IR spectral data of ligands and their metal complexes $\left(\mathrm{cm}^{-1}\right)$.

\begin{tabular}{|c|c|c|c|c|c|c|c|}
\hline Compound & $\nu(\mathrm{OH})$ & $\nu(\mathrm{C}=\mathrm{O})(\gamma$ pyrone $)$ & $\nu(\mathrm{C}=\mathrm{N})$ & $\nu(\mathrm{CO})$ (carboxylate) & $v(\mathrm{C}-\mathrm{S})$ & $\nu(\mathrm{M}-\mathrm{N})$ & $v(\mathrm{M}-\mathrm{O})$ \\
\hline $\mathrm{HL}_{1}$ & 3241 & 1643 & 1605 & & & & \\
\hline $\mathrm{HL}_{2}$ & & 1651 & 1605 & 1365 & & & \\
\hline $\mathrm{HL}_{3}$ & 3246 & 1647 & 1591 & & & & \\
\hline $\mathrm{HL}_{4}$ & & 1622 & 1597 & & 790 & & \\
\hline$\left[\mathrm{Ni}\left(\mathrm{L}_{1}\right)_{2}\right]$ & & 1619 & 1563 & & & 467 & 586 \\
\hline$\left[\mathrm{Ni}\left(\mathrm{L}_{2}\right)_{2}\right] \cdot \mathrm{H}_{2} \mathrm{O}$ & & 1618 & 1570 & 1318 & & 419 & 514 \\
\hline$\left[\mathrm{Ni}\left(\mathrm{L}_{3}\right)_{2}\right] \cdot \mathrm{H}_{2} \mathrm{O}$ & & 1615 & 1563 & & & 480 & 500 \\
\hline$\left[\mathrm{Ni}\left(\mathrm{L}_{4}\right)_{2}\right]$ & & 1598 & 1564 & & 743 & 443 & 524 \\
\hline$\left[\mathrm{Zn}\left(\mathrm{L}_{1}\right)_{2}\right] \cdot \mathrm{H}_{2} \mathrm{O}$ & & 1614 & 1574 & & & 460 & 599 \\
\hline$\left[\mathrm{Zn}\left(\mathrm{L}_{2}\right)_{2}\right]$ & & 1615 & 1563 & 1347 & & 480 & 500 \\
\hline$\left[\mathrm{Zn}\left(\mathrm{L}_{3}\right)_{2}\right]$ & & 1615 & 1563 & & & 488 & 521 \\
\hline$\left[\mathrm{Zn}\left(\mathrm{L}_{4}\right)_{2}\right] \cdot \mathrm{H}_{2} \mathrm{O}$ & & 1609 & 1581 & & 751 & 441 & 505 \\
\hline
\end{tabular}

$\beta=116.71^{\circ}, \gamma=90.01^{\circ}$, and $V=562(\AA)^{3} ;\left[\mathrm{Ni}\left(\mathrm{L}_{2}\right)_{2}\right] \cdot \mathrm{H}_{2} \mathrm{O}$ : $a=8.24 \AA, b=9.24 \AA, c=7.32 \AA, \alpha=104.12^{\circ}, \beta=124.44^{\circ}$, $\gamma=98.04^{\circ}$, and $V=557(\AA)^{3} ;\left[\mathrm{Ni}\left(\mathrm{L}_{3}\right)_{2}\right] \cdot \mathrm{H}_{2} \mathrm{O}: a=9.40 \AA$, $b=9.20 \AA, c=8.46 \AA, \alpha=97.45^{\circ}, \beta=107.38^{\circ}, \gamma=91.45^{\circ}$, and $V=731(\AA)^{3} ;\left[\mathrm{Ni}\left(\mathrm{L}_{4}\right)_{2}\right]: a=7.73 \AA, b=8.24 \AA$, $c=8.49 \AA, \alpha=98.30^{\circ}, \beta=104.76^{\circ}, \gamma=90.55^{\circ}$, and $V=540(\AA)^{3} ;\left[\mathrm{Zn}\left(\mathrm{L}_{1}\right)_{2}\right] \cdot \mathrm{H}_{2} \mathrm{O}: a=9.60 \AA, b=9.94 \AA$, $c=8.89 \AA, \alpha=102.34^{\circ}, \beta=109.69^{\circ}, \gamma=93.94^{\circ}$, and $V=848(\AA)^{3} ;\left[\mathrm{Zn}\left(\mathrm{L}_{3}\right)_{2}\right]: a=8.76 \AA, b=9.04 \AA, c=8.61 \AA$, $\alpha=105.78^{\circ}, \beta=103.02^{\circ}, \gamma=90.21^{\circ}$, and $V=682(\AA)^{3}$. The average crystallite sizes $(D)$ of the metal complexes are calculated from the Scherrer formula [41]:

$$
D=\frac{0.9 \lambda}{\beta \cos \theta},
$$

where $\lambda$ is the $\mathrm{X}$-ray wavelength, $\beta$ is the full width at half maximum of prominent intensity peak, and $\theta$ is the diffraction angle. The $\left[\mathrm{Ni}\left(\mathrm{L}_{1}\right)_{2}\right],\left[\mathrm{Ni}\left(\mathrm{L}_{2}\right)_{2}\right] \cdot \mathrm{H}_{2} \mathrm{O},\left[\mathrm{Ni}\left(\mathrm{L}_{3}\right)_{2}\right] \cdot \mathrm{H}_{2} \mathrm{O}$, $\left[\mathrm{Ni}\left(\mathrm{L}_{4}\right)_{2}\right],\left[\mathrm{Zn}\left(\mathrm{L}_{1}\right)_{2}\right] \cdot \mathrm{H}_{2} \mathrm{O}$, and $\left[\mathrm{Zn}\left(\mathrm{L}_{3}\right)_{2}\right]$ complexes have an average crystallite size of $70,17,16,27,42$, and $53 \mathrm{~nm}$, respectively, suggesting that the complexes are in nanocrystalline phase.

The SEM (Scanning Electron Microscope) is used to evaluate the morphology and particle size of the compounds. The SEM photographs of $\left[\mathrm{Ni}\left(\mathrm{L}_{3}\right)_{2}\right] \cdot \mathrm{H}_{2} \mathrm{O}$ and $\left[\mathrm{Ni}\left(\mathrm{L}_{4}\right)_{2}\right]$ are shown in Figure 6. The SEM micrographs show the agglomerate particles of the complexes. In case of $\left[\mathrm{Ni}\left(\mathrm{L}_{3}\right)_{2}\right] \cdot \mathrm{H}_{2} \mathrm{O}$ and $\left[\mathrm{Ni}\left(\mathrm{L}_{4}\right)_{2}\right]$ complexes, some agglomerates appear to have tiny needles, while the other agglomerates appear to be of spherical plates like morphologies. 
TABLE 3: Electronic, magnetic, and ligand field parameters of $\mathrm{Ni}(\mathrm{II})$ complexes.

\begin{tabular}{|c|c|c|c|c|c|c|c|c|}
\hline Compound & $\begin{array}{c}\text { Absorption maxima } \\
\left(\mathrm{cm}^{-1}\right)\end{array}$ & Tentative assignments & $\begin{array}{c}\text { Magnetic moment } \\
\text { (B.M) }\end{array}$ & $v_{2} / \nu_{1}$ & $\begin{array}{l}10 \mathrm{Dq} \\
\left(\mathrm{cm}^{-1}\right)\end{array}$ & $\begin{array}{c}\mathrm{B} \\
\left(\mathrm{cm}^{-1}\right)\end{array}$ & $\beta$ & $\begin{array}{c}\text { LFSE } \\
\left(\mathrm{kJ} \cdot \mathrm{mol}^{-1}\right)\end{array}$ \\
\hline$\left[\mathrm{Ni}\left(\mathrm{L}_{1}\right)_{2}\right]$ & $\begin{array}{c}8532 \\
15200 \\
24900 \\
\end{array}$ & $\begin{array}{l}{ }^{3} \mathrm{~A}_{2 \mathrm{~g}}(\mathrm{~F}) \rightarrow{ }^{3} \mathrm{~T}_{2 \mathrm{~g}}(\mathrm{~F})\left(v_{1}\right) \\
{ }^{3} \mathrm{~A}_{2 \mathrm{~g}}(\mathrm{~F}) \rightarrow{ }^{3} \mathrm{~T}_{1 \mathrm{~g}}(\mathrm{~F})\left(v_{2}\right) \\
{ }^{3} \mathrm{~A}_{2 \mathrm{~g}}(\mathrm{~F}) \rightarrow{ }^{3} \mathrm{~T}_{1 \mathrm{~g}}(\mathrm{P})\left(v_{3}\right)\end{array}$ & 3.12 & 1.78 & 8532 & 966 & 0.92 & 122.50 \\
\hline$\left[\mathrm{Ni}\left(\mathrm{L}_{2}\right)_{2}\right] \cdot \mathrm{H}_{2} \mathrm{O}$ & $\begin{array}{c}8787 \\
16000 \\
24390 \\
\end{array}$ & $\begin{array}{l}{ }^{3} \mathrm{~A}_{2 \mathrm{~g}}(\mathrm{~F}) \rightarrow{ }^{3} \mathrm{~T}_{2 \mathrm{~g}}(\mathrm{~F})\left(v_{1}\right) \\
{ }^{3} \mathrm{~A}_{2 \mathrm{~g}}(\mathrm{~F}) \rightarrow{ }^{3} \mathrm{~T}_{1 \mathrm{~g}}(\mathrm{~F})\left(v_{2}\right) \\
{ }^{3} \mathrm{~A}_{2 \mathrm{~g}}(\mathrm{~F}) \rightarrow{ }^{3} \mathrm{~T}_{1 \mathrm{~g}}(\mathrm{P})\left(v_{3}\right)\end{array}$ & 3.18 & 1.82 & 8787 & 935 & 0.89 & 126.19 \\
\hline$\left[\mathrm{Ni}\left(\mathrm{L}_{3}\right)_{2}\right] \cdot \mathrm{H}_{2} \mathrm{O}$ & $\begin{array}{c}8313 \\
14705 \\
20833 \\
24390\end{array}$ & $\begin{array}{l}{ }^{3} \mathrm{~A}_{2 \mathrm{~g}}(\mathrm{~F}) \rightarrow{ }^{3} \mathrm{~T}_{2 \mathrm{~g}}(\mathrm{~F})\left(v_{1}\right) \\
{ }^{3} \mathrm{~A}_{2 \mathrm{~g}}(\mathrm{~F}) \rightarrow{ }^{3} \mathrm{~T}_{1 \mathrm{~g}}(\mathrm{~F})\left(v_{2}\right) \\
{ }^{3} \mathrm{~A}_{2 \mathrm{~g}}(\mathrm{~F}) \rightarrow{ }^{3} \mathrm{~T}_{1 \mathrm{~g}}(\mathrm{P})\left(v_{3}\right)\end{array}$ & 3.25 & 1.76 & 8313 & 943 & 0.90 & 119.40 \\
\hline$\left[\mathrm{Ni}\left(\mathrm{L}_{4}\right)_{2}\right]$ & $\begin{array}{l}8628 \\
16313 \\
24570\end{array}$ & $\begin{array}{l}{ }^{3} \mathrm{~A}_{2 \mathrm{~g}}(\mathrm{~F}) \rightarrow{ }^{3} \mathrm{~T}_{2 \mathrm{~g}}(\mathrm{~F})\left(v_{1}\right) \\
{ }^{3} \mathrm{~A}_{2 \mathrm{~g}}(\mathrm{~F}) \rightarrow{ }^{3} \mathrm{~T}_{1 \mathrm{~g}}(\mathrm{~F})\left(v_{2}\right) \\
{ }^{3} \mathrm{~A}_{2 \mathrm{~g}}(\mathrm{~F}) \rightarrow{ }^{3} \mathrm{~T}_{1 \mathrm{~g}}(\mathrm{P})\left(v_{3}\right)\end{array}$ & 2.91 & 1.89 & 8628 & 999 & 0.95 & 123.92 \\
\hline
\end{tabular}

TABLE 4: Thermal analysis results of metal complexes.

\begin{tabular}{|c|c|c|c|}
\hline Compound & Temperature $\left({ }^{\circ} \mathrm{C}\right)$ & Found (cald.) & Assignment \\
\hline \multirow{3}{*}{$\begin{array}{l}{\left[\mathrm{Ni}\left(\mathrm{L}_{1}\right)_{2}\right]} \\
{\left[\mathrm{Ni}\left(\mathrm{C}_{16} \mathrm{H}_{10} \mathrm{O}_{3} \mathrm{~N}\right)_{2}\right]}\end{array}$} & $180-420$ & $19.38(18.36)$ & $\mathrm{C}_{6} \mathrm{H}_{4} \mathrm{ON}$ \\
\hline & $421-718$ & $68.55(69.02)$ & $\mathrm{C}_{26} \mathrm{H}_{16} \mathrm{O}_{5} \mathrm{~N}$ \\
\hline & $>718$ & $12.07(12.62)$ & $\mathrm{NiO}$ \\
\hline \multirow{4}{*}{$\begin{array}{l}{\left[\mathrm{Ni}\left(\mathrm{L}_{2}\right)_{2}\right] \cdot \mathrm{H}_{2} \mathrm{O}} \\
{\left[\mathrm{Ni}\left(\mathrm{C}_{17} \mathrm{H}_{10} \mathrm{O}_{4} \mathrm{~N}\right)_{2}\right] \cdot \mathrm{H}_{2} \mathrm{O}}\end{array}$} & $30-90$ & $2.83(2.72)$ & $\mathrm{H}_{2} \mathrm{O}$ \\
\hline & $335-478$ & $60.71(62.05)$ & $\mathrm{C}_{24} \mathrm{H}_{14} \mathrm{O}_{5} \mathrm{~N}_{2}$ \\
\hline & $479-849$ & $23.93(23.61)$ & $\mathrm{C}_{10} \mathrm{H}_{6} \mathrm{O}_{2}$ \\
\hline & $>849$ & $12.53(11.62)$ & $\mathrm{NiO}$ \\
\hline \multirow{3}{*}{$\begin{array}{l}{\left[\mathrm{Ni}\left(\mathrm{L}_{3}\right)_{2}\right] \cdot \mathrm{H}_{2} \mathrm{O}} \\
{\left[\mathrm{Ni}\left(\mathrm{C}_{15} \mathrm{H}_{9} \mathrm{O}_{3} \mathrm{~N}_{2}\right)_{2}\right] \cdot \mathrm{H}_{2} \mathrm{O}}\end{array}$} & $30-65$ & $3.09(2.96)$ & $\mathrm{H}_{2} \mathrm{O}$ \\
\hline & $180-879$ & $83.40(84.73)$ & $\mathrm{C}_{30} \mathrm{H}_{18} \mathrm{O}_{5} \mathrm{~N}_{4}$ \\
\hline & $>880$ & $13.51(12.31)$ & $\mathrm{NiO}$ \\
\hline \multirow{3}{*}{$\begin{array}{l}{\left[\mathrm{Ni}\left(\mathrm{L}_{4}\right)_{2}\right]} \\
{\left[\mathrm{Ni}\left(\mathrm{C}_{16} \mathrm{H}_{10} \mathrm{O}_{2} \mathrm{NS}\right)_{2}\right]}\end{array}$} & $171-410$ & $45.91(45.25)$ & $\mathrm{C}_{16} \mathrm{H}_{10} \mathrm{O}_{2} \mathrm{NS}$ \\
\hline & $411-670$ & $42.14(42.67)$ & $\mathrm{C}_{16} \mathrm{H}_{10} \mathrm{ONS}$ \\
\hline & $>670$ & 11.95 (12.07) & $\mathrm{NiO}$ \\
\hline \multirow{4}{*}{$\begin{array}{l}{\left[\mathrm{Zn}\left(\mathrm{L}_{1}\right)_{2}\right] \cdot \mathrm{H}_{2} \mathrm{O}} \\
{\left[\mathrm{Zn}\left(\mathrm{C}_{16} \mathrm{H}_{10} \mathrm{O}_{3} \mathrm{~N}\right)_{2}\right] \cdot \mathrm{H}_{2} \mathrm{O}}\end{array}$} & $30-135$ & $2.45(2.95)$ & $\mathrm{H}_{2} \mathrm{O}$ \\
\hline & $194-422$ & $24.95(25.92)$ & $\mathrm{C}_{10} \mathrm{H}_{6} \mathrm{O}_{2}$ \\
\hline & $423-790$ & $59.48(58.09)$ & $\mathrm{C}_{22} \mathrm{H}_{14} \mathrm{O}_{3} \mathrm{~N}_{2}$ \\
\hline & $>791$ & $13.12(13.04)$ & $\mathrm{ZnO}$ \\
\hline \multirow{3}{*}{$\begin{array}{l}{\left[\mathrm{Zn}\left(\mathrm{L}_{2}\right)_{2}\right]} \\
{\left[\mathrm{Zn}\left(\mathrm{C}_{17} \mathrm{H}_{10} \mathrm{O}_{4} \mathrm{~N}\right)_{2}\right]}\end{array}$} & $195-265$ & $15.55(16.01)$ & $\mathrm{C}_{7} \mathrm{H}_{4} \mathrm{O}$ \\
\hline & $266-661$ & $69.76(71.46)$ & $\mathrm{C}_{27} \mathrm{H}_{16} \mathrm{O}_{4} \mathrm{~N}_{2}$ \\
\hline & $>662$ & $14.69(12.53)$ & $\mathrm{ZnO}$ \\
\hline \multirow{4}{*}{$\begin{array}{l}{\left[\mathrm{Zn}\left(\mathrm{L}_{3}\right)_{2}\right]} \\
{\left[\mathrm{Zn}\left(\mathrm{C}_{15} \mathrm{H}_{9} \mathrm{O}_{3} \mathrm{~N}_{2}\right)_{2}\right]}\end{array}$} & $157-192$ & $10.91(12.93)$ & $\mathrm{C}_{5} \mathrm{H}_{3} \mathrm{~N}$ \\
\hline & $193-485$ & $28.65(29.22)$ & $\mathrm{C}_{10} \mathrm{H}_{6} \mathrm{O}_{2} \mathrm{~N}$ \\
\hline & $486-835$ & 46.03 (44.18) & $\mathrm{C}_{15} \mathrm{H}_{9} \mathrm{O}_{3} \mathrm{~N}_{2}$ \\
\hline & $>836$ & $14.41(13.67)$ & $\mathrm{ZnO}$ \\
\hline \multirow{4}{*}{$\begin{array}{l}{\left[\mathrm{Zn}\left(\mathrm{L}_{4}\right)_{2}\right] \cdot \mathrm{H}_{2} \mathrm{O}} \\
{\left[\mathrm{Zn}\left(\mathrm{C}_{16} \mathrm{H}_{10} \mathrm{O}_{2} \mathrm{NS}\right)_{2}\right] \cdot \mathrm{H}_{2} \mathrm{O}}\end{array}$} & $30-124$ & $2.41(2.79)$ & $\mathrm{H}_{2} \mathrm{O}$ \\
\hline & $165-348$ & $27.41(26.73)$ & $\mathrm{C}_{10} \mathrm{H}_{6} \mathrm{O}_{2} \mathrm{~N}$ \\
\hline & $389-632$ & $56.98(57.84)$ & $\mathrm{C}_{22} \mathrm{H}_{14} \mathrm{ONS}_{2}$ \\
\hline & $>632$ & $13.20(12.64)$ & $\mathrm{ZnO}$ \\
\hline
\end{tabular}


TABLE 5: Observed and calculated powder XRD patterns of (a) $\left[\mathrm{Ni}\left(\mathrm{L}_{1}\right)_{2}\right]$ (b) $\left[\mathrm{Zn}\left(\mathrm{L}_{1}\right)_{2}\right] \cdot \mathrm{H}_{2} \mathrm{O}$.

(a)

\begin{tabular}{|c|c|c|c|c|c|c|c|c|}
\hline \multirow{2}{*}{ S. number } & \multicolumn{2}{|c|}{$2 \theta$} & \multirow{2}{*}{$\Delta 2 \theta$} & \multicolumn{2}{|c|}{$d$-spacing } & \multirow{2}{*}{$h$} & \multirow{2}{*}{$k$} & \multirow{2}{*}{$l$} \\
\hline & Observed & Calculated & & Observed & Calculated & & & \\
\hline 1 & 10.57 & 10.57 & 0 & 8.3627 & 8.3627 & 0 & 1 & 0 \\
\hline 2 & 11.29 & 11.29 & 0 & 7.8314 & 7.8314 & 1 & 0 & 0 \\
\hline 3 & 13.44 & 13.44 & 0 & 6.5805 & 6.5805 & 0 & 0 & 1 \\
\hline 4 & 14.79 & 14.79 & 0 & 5.9847 & 5.9847 & -1 & 1 & 0 \\
\hline 5 & 15.75 & 15.75 & 0 & 5.6205 & 5.6205 & -1 & -1 & 1 \\
\hline 6 & 17.79 & 17.79 & 0 & 4.9805 & 4.9805 & -1 & 1 & 1 \\
\hline 7 & 18.85 & 18.68 & 0.164 & 4.7046 & 4.7455 & 0 & 1 & 1 \\
\hline 8 & 21.16 & 21.22 & -0.06 & 4.1962 & 4.1845 & 1 & 0 & 1 \\
\hline 9 & 22.14 & 22.06 & 0.074 & 4.0121 & 4.0255 & 1 & -1 & 1 \\
\hline 10 & 24.05 & 24.07 & -0.013 & 3.6968 & 3.6948 & -1 & 0 & 2 \\
\hline 11 & 25.04 & 24.99 & 0.054 & 3.5533 & 3.5608 & 1 & 2 & 0 \\
\hline 12 & 26.33 & 26.35 & -0.017 & 3.382 & 3.3799 & -1 & 2 & 1 \\
\hline 13 & 28.1 & 28.04 & 0.061 & 3.1731 & 3.1799 & -1 & 1 & 2 \\
\hline 14 & 32.65 & 32.65 & 0 & 2.7404 & 2.7405 & 2 & 2 & 0 \\
\hline 15 & 35.06 & 35.07 & -0.012 & 2.5575 & 2.5566 & -3 & 1 & 0 \\
\hline 16 & 38.92 & 38.86 & 0.054 & 2.3124 & 2.3155 & -2 & 3 & 1 \\
\hline 17 & 43.89 & 43.95 & -0.058 & 2.0612 & 2.0586 & -1 & 3 & 2 \\
\hline 18 & 45.13 & 45.16 & -0.033 & 2.0076 & 2.0062 & -2 & -3 & 3 \\
\hline
\end{tabular}

(b)

\begin{tabular}{|c|c|c|c|c|c|c|c|c|}
\hline \multirow{2}{*}{ S. number } & \multicolumn{2}{|c|}{$2 \theta$ values } & \multirow{2}{*}{$\Delta 2 \theta$} & \multicolumn{2}{|c|}{$d$-spacing } & \multirow{2}{*}{$h$} & \multirow{2}{*}{$k$} & \multirow{2}{*}{$l$} \\
\hline & Observed & Calculated & & Observed & Calculated & & & \\
\hline 1 & 12.58 & 12.58 & 0 & 7.0315 & 7.0315 & 1 & 1 & 0 \\
\hline 2 & 13.81 & 13.81 & 0 & 6.4077 & 6.4077 & -1 & 0 & 1 \\
\hline 3 & 15.73 & 15.73 & 0 & 5.6299 & 5.6299 & 1 & 0 & 1 \\
\hline 4 & 17.35 & 17.35 & 0 & 5.1064 & 5.1064 & -1 & 1 & 1 \\
\hline 5 & 20.75 & 20.77 & -0.017 & 4.2777 & 4.2742 & -1 & 3 & 0 \\
\hline 6 & 24.09 & 24.13 & -0.031 & 3.6907 & 3.6859 & -1 & -3 & 2 \\
\hline 7 & 25.32 & 25.31 & 0.012 & 3.5141 & 3.5158 & 2 & 2 & 0 \\
\hline 8 & 27.93 & 27.97 & -0.038 & 3.1916 & 3.1873 & -1 & -4 & 2 \\
\hline 9 & 30.15 & 30.16 & -0.008 & 2.9620 & 2.9612 & 0 & -3 & 3 \\
\hline 10 & 31.92 & 31.90 & 0.021 & 2.8015 & 2.8033 & 2 & -5 & 2 \\
\hline 11 & 34.43 & 34.45 & -0.017 & 2.6028 & 2.6016 & -1 & 5 & 0 \\
\hline 12 & 36.05 & 36.06 & -0.008 & 2.4892 & 2.4886 & -1 & 0 & 3 \\
\hline 13 & 42.75 & 42.83 & -0.081 & 2.1137 & 2.1099 & 3 & 1 & 2 \\
\hline
\end{tabular}

3.6. Fluorescence Spectra. The fluorescence characteristics of metal complexes were studied at room temperature in solid state. In metal complexes metal to ligand coordination may lead to significant changes of the fluorescence properties of the ligand, including increase or decrease of the intensity, emission wavelength shift, quenching of the fluorescence, or appearance of new emissions [42]. The fluorescence spectra of $\mathrm{HL}_{1}$ ligand and its metal (Ni(II) and $\mathrm{Zn}(\mathrm{II})$ ) complexes were depicted in Figure 7. The $\mathrm{Ni}$ (II) and $\mathrm{Zn}$ (II) complexes of $\mathrm{HL}_{1}$ were characterised by emission bands around (522, $569) \mathrm{nm}$ and $(458,644) \mathrm{nm}$, upon photo excitation at 453 and $357 \mathrm{~nm}$, respectively. The $\mathrm{Ni}(\mathrm{II})$ complex of $\mathrm{HL}_{2}$ exhibits emission bands at 449 and $524 \mathrm{~nm}$. $\mathrm{Zn}$ (II) complex of $\mathrm{HL}_{2}$ does not show any emission spectra. The $\mathrm{Ni}$ (II) and $\mathrm{Zn}$ (II) complexes of $\mathrm{HL}_{3}$ were characterised by an emission bands at $(504,570$, and 668$)$ and $419 \mathrm{~nm}$, respectively. The $\mathrm{Ni}(\mathrm{II})$ and $\mathrm{Zn}$ (II) complexes of $\mathrm{HL}_{4}$ exhibit emission bands around 539 and $613 \mathrm{~nm}$, respectively. From the results, red shift and quenching of the metal ions was observed in the case of ligands and its metal complexes.

3.7. Antimicrobial Activity. The minimum inhibitory concentrations (MIC) of the complexes compared with the ligands and standard drugs are listed in Table 6 . The results indicate that the metal complexes displayed more antibacterial activity compared to the parent ligands under similar experimental 
TABLE 6: MIC values of antimicrobial activity of ligands and their metal complexes $(\mu \mathrm{g} / \mathrm{mL})$.

\begin{tabular}{|c|c|c|c|c|c|}
\hline Compound & Bacillus subtilis & Staphylococcus aureus & Proteus vulgaris & Klebsiella pneumoniae & Candida albicans \\
\hline $\mathrm{HL}_{1}$ & - & - & - & - & - \\
\hline $\mathrm{HL}_{2}$ & 80 & 80 & 80 & 80 & 80 \\
\hline $\mathrm{HL}_{3}$ & - & - & - & - & - \\
\hline $\mathrm{HL}_{4}$ & - & - & - & - & - \\
\hline$\left[\mathrm{Ni}\left(\mathrm{L}_{1}\right)_{2}\right]$ & 35 & 40 & 32 & 41 & 75 \\
\hline$\left[\mathrm{Ni}\left(\mathrm{L}_{2}\right)_{2}\right] \cdot \mathrm{H}_{2} \mathrm{O}$ & 70 & 80 & 65 & 85 & - \\
\hline$\left[\mathrm{Ni}\left(\mathrm{L}_{3}\right)_{2}\right] \cdot \mathrm{H}_{2} \mathrm{O}$ & 45 & 45 & 50 & 33 & 33 \\
\hline$\left[\mathrm{Ni}\left(\mathrm{L}_{4}\right)_{2}\right]$ & 75 & 80 & - & 78 & - \\
\hline$\left[\mathrm{Zn}\left(\mathrm{L}_{1}\right)_{2}\right] \cdot \mathrm{H}_{2} \mathrm{O}$ & - & - & - & - & - \\
\hline$\left[\mathrm{Zn}\left(\mathrm{L}_{2}\right)_{2}\right]$ & 85 & 78 & 80 & 75 & 80 \\
\hline$\left[\mathrm{Zn}\left(\mathrm{L}_{3}\right)_{2}\right]$ & 30 & 30 & 33 & 30 & 33 \\
\hline$\left[\mathrm{Zn}\left(\mathrm{L}_{4}\right)_{2}\right] \cdot \mathrm{H}_{2} \mathrm{O}$ & - & 80 & - & 80 & - \\
\hline Kanamycin & 4 & 10 & 8 & 11 & - \\
\hline Clotrimazole & - & - & - & - & 10 \\
\hline
\end{tabular}

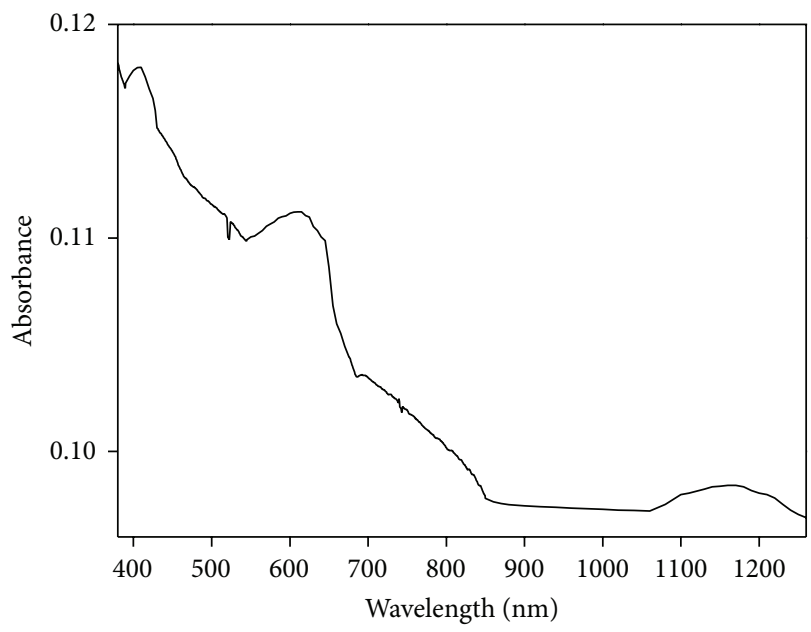

(a)

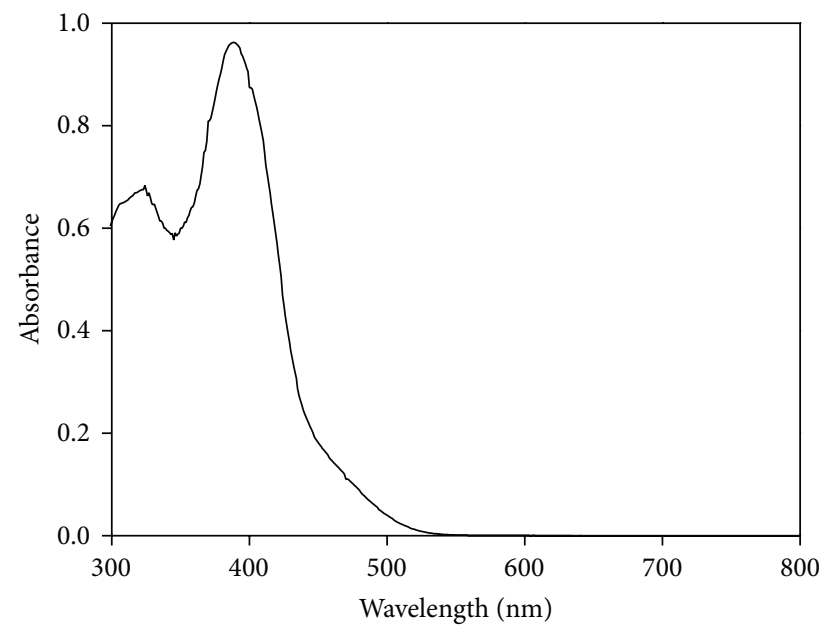

(b)

FIgURE 2: Electronic spectra of (a) $\left[\mathrm{Ni}\left(\mathrm{L}_{4}\right)_{2}\right]$ and $(\mathrm{b})\left[\mathrm{Zn}\left(\mathrm{L}_{4}\right)_{2}\right] \cdot \mathrm{H}_{2} \mathrm{O}$.

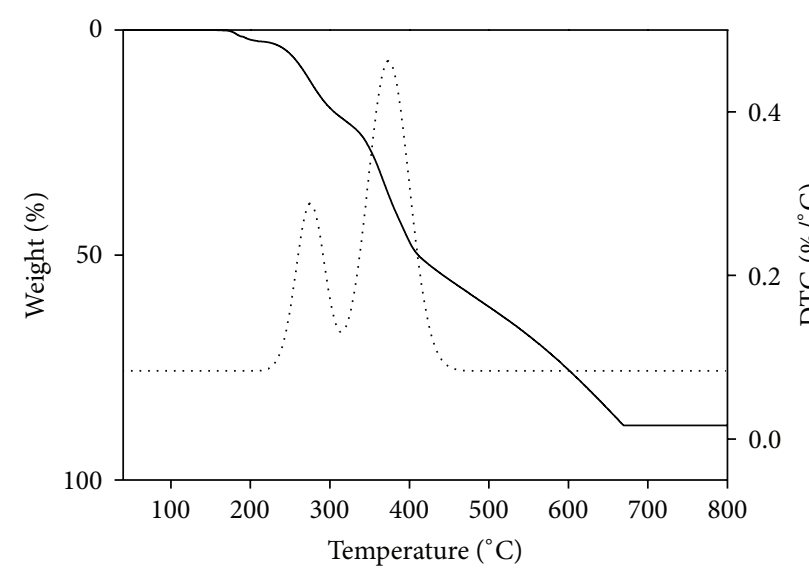

(a)

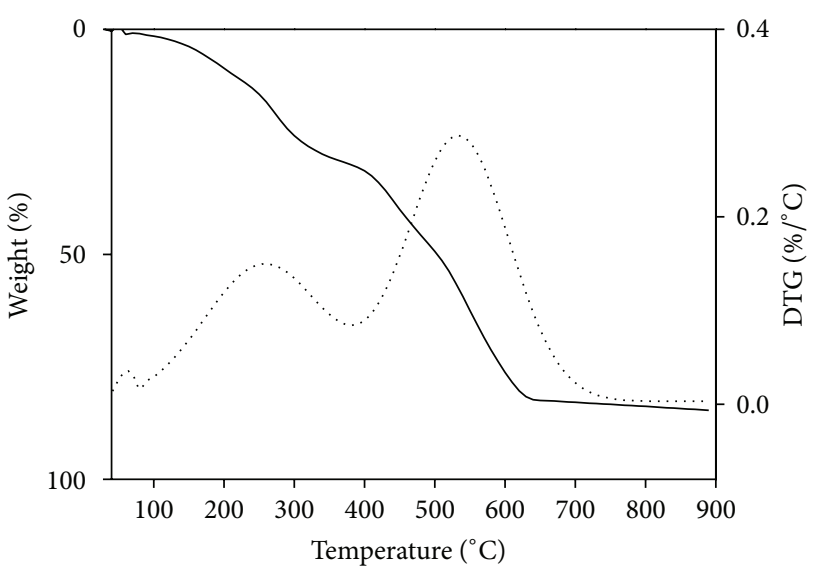

(b)

FIGURE 3: TG-DTG graph of (a) $\left[\mathrm{Ni}\left(\mathrm{L}_{4}\right)_{2}\right]$ and (b) $\left[\mathrm{Zn}\left(\mathrm{L}_{4}\right)_{2}\right] \cdot \mathrm{H}_{2} \mathrm{O}$. 


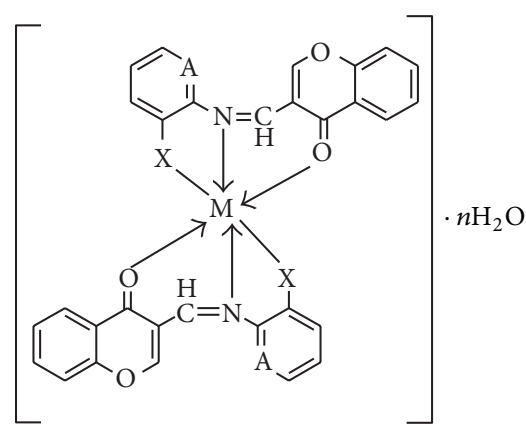

$\mathrm{A}=\mathrm{C}$ and $\mathrm{X}=\mathrm{O} / \mathrm{S} / \mathrm{COO}$

$\mathrm{A}=\mathrm{N}$ and $\mathrm{X}=\mathrm{O}$

$M=N i(I I) / Z n(I I)$

FIgURE 4: Proposed structure of the metal complexes.

conditions on same microorganisms except $\left[\mathrm{Ni}\left(\mathrm{L}_{2}\right)_{2}\right] \cdot \mathrm{H}_{2} \mathrm{O}$ and $\left[\mathrm{Zn}\left(\mathrm{L}_{1}\right)_{2}\right] \cdot \mathrm{H}_{2} \mathrm{O}$ complexes. The results indicate that the metal complexes displayed more antibacterial activity compared to the parent ligands under similar experimental conditions on same microorganisms except $\left[\mathrm{Zn}\left(\mathrm{L}_{1}\right)_{2}\right] \cdot \mathrm{H}_{2} \mathrm{O}$. However, $\left[\mathrm{Ni}\left(\mathrm{L}_{1}\right)_{2}\right],\left[\mathrm{Ni}\left(\mathrm{L}_{3}\right)_{2}\right] \cdot \mathrm{H}_{2} \mathrm{O},\left[\mathrm{Zn}\left(\mathrm{L}_{2}\right)_{2}\right]$ and $\left[\mathrm{Zn}\left(\mathrm{L}_{3}\right)_{2}\right]$ complexes showed antifungal activity while the remaining complexes did not show any activity. However, $\left[\mathrm{Ni}\left(\mathrm{L}_{1}\right)_{2}\right]$, $\left[\mathrm{Ni}\left(\mathrm{L}_{3}\right)_{2}\right] \cdot \mathrm{H}_{2} \mathrm{O}$, and $\left[\mathrm{Zn}\left(\mathrm{L}_{3}\right)_{2}\right]$ complexes showed antifungal activity while the remaining complexes did not show any activity. Among all the metal complexes $\left[\mathrm{Zn}\left(\mathrm{L}_{3}\right)_{2}\right]$ complex showed good activity against all bacteria and fungi strains. Increase in the activity of the complexes compared to that of ligands can be explained on the basis of Overtone's concept and Tweedy's chelation theory. The theory states that the polarity of the metal ion is reduced on complexation due to the partial sharing of its positive charge with donor groups. Consequently, the positive charge is delocalized over the whole ring, which causes the improved lipophilicity of the compound through cell membrane of the pathogen [43]. The negative results can be attributed either to the inability of the complexes to diffuse into the bacteria cell membrane and hence they become unable to interfere with its biological activity or they can diffuse and become inactivated by unknown cellular mechanism, that is, bacterial enzymes [44].

3.8. Nematicidal Activity. All complexes except $\left[\mathrm{Ni}\left(\mathrm{L}_{3}\right)_{2}\right]$. $\mathrm{H}_{2} \mathrm{O}$ and $\left[\mathrm{Zn}\left(\mathrm{L}_{3}\right)_{2}\right]$ showed very less nematicidal activity against Meloidogyne incognita. However, $\left[\mathrm{Ni}\left(\mathrm{L}_{3}\right)_{2}\right] \cdot \mathrm{H}_{2} \mathrm{O}$ and $\left[\mathrm{Zn}\left(\mathrm{L}_{3}\right)_{2}\right]$ complexes exhibited moderate activity indicating $47 \%$ and $51 \%$ mortality, respectively, after $48 \mathrm{~h}$ exposure in $250 \mu \mathrm{g} / \mathrm{mL}$ concentration. However, the activity of the metal complexes depends on concentration and time, that is, activity was higher at high concentrations and increased with time.

3.9. DPPH Radical Scavenging Activity. The antioxidant activity of the complexes was tested by measuring their ability to donate an electron to the free radical compound
TABLE 7: $\mathrm{IC}_{50}$ values $(\mu \mathrm{g} / \mathrm{mL})$ of DPPH radical scavenging activity of ligands and their metal complexes.

\begin{tabular}{lc}
\hline Compound & $\mathrm{IC}_{50}(\mu \mathrm{g} / \mathrm{mL})$ \\
\hline $\mathrm{HL}_{3}$ & 1.27 \\
$\mathrm{HL}_{4}$ & 0.40 \\
{$\left[\mathrm{Ni}\left(\mathrm{L}_{1}\right)_{2}\right]$} & 1.21 \\
{$\left[\mathrm{Ni}\left(\mathrm{L}_{4}\right)_{2}\right]$} & 1.09 \\
{$\left[\mathrm{Zn}\left(\mathrm{L}_{4}\right)_{2}\right] \cdot \mathrm{H}_{2} \mathrm{O}$} & 0.69 \\
$\mathrm{BHT}$ & 0.67 \\
\hline
\end{tabular}

TABLE 8: $\mathrm{IC}_{50}$ values $(\mu \mathrm{g} / \mathrm{mL})$ of cytotoxic activity of ligands and their metal complexes.

\begin{tabular}{lccc}
\hline Compound & Raw & MCF-7 & COLO 205 \\
\hline $\mathrm{HL}_{1}$ & 46.8 & 24.5 & 20.1 \\
$\mathrm{HL}_{2}$ & 56.1 & 34.2 & 39.1 \\
$\mathrm{HL}_{3}$ & 52.8 & 29.2 & 15.2 \\
$\mathrm{HL}_{4}$ & 46.9 & 30.4 & 68.1 \\
{$\left[\mathrm{Ni}\left(\mathrm{L}_{1}\right)_{2}\right]$} & 224.8 & 67.1 & 54.6 \\
{$\left[\mathrm{Ni}\left(\mathrm{L}_{2}\right)_{2}\right] \cdot \mathrm{H}_{2} \mathrm{O}$} & 36.3 & 26.5 & 60.9 \\
{$\left[\mathrm{Ni}\left(\mathrm{L}_{3}\right)_{2}\right] \cdot \mathrm{H}_{2} \mathrm{O}$} & 53.0 & 36.9 & 71.4 \\
{$\left[\mathrm{Ni}\left(\mathrm{L}_{4}\right)_{2}\right]$} & 58.6 & 35.6 & 49.9 \\
{$\left[\mathrm{Zn}\left(\mathrm{L}_{1}\right)_{2}\right] \cdot \mathrm{H}_{2} \mathrm{O}$} & 44.0 & 40.1 & 54.7 \\
{$\left[\mathrm{Zn}\left(\mathrm{L}_{2}\right)_{2}\right]$} & 34.0 & 52.3 & 43.9 \\
{$\left[\mathrm{Zn}\left(\mathrm{L}_{3}\right)_{2}\right]$} & 33.1 & 22.6 & 42.0 \\
{$\left[\mathrm{Zn}\left(\mathrm{L}_{4}\right)_{2}\right] \cdot \mathrm{H}_{2} \mathrm{O}$} & 42.5 & 39.2 & 53.5 \\
$\mathrm{Cis}-$ platin & 1.5 & 1.7 & 5.6 \\
\hline
\end{tabular}

DPPH, monitoring changes in absorption at $517 \mathrm{~nm}$ using UV-VIS spectrophotometer. $\mathrm{IC}_{50}$ values were calculated and compared with the standard. $\mathrm{IC}_{50}$ values of the metal complexes are tabulated in Table 7. All complexes showed very less activity except $\left[\mathrm{Ni}\left(\mathrm{L}_{1}\right)_{2}\right],\left[\mathrm{Ni}\left(\mathrm{L}_{4}\right)_{2}\right]$, and $\left[\mathrm{Zn}\left(\mathrm{L}_{4}\right)_{2}\right] \cdot \mathrm{H}_{2} \mathrm{O}$ complexes. Among all the complexes $\left[\mathrm{Zn}\left(\mathrm{L}_{4}\right)_{2}\right] \cdot \mathrm{H}_{2} \mathrm{O}\left(\mathrm{IC}_{50}=\right.$ $0.69 \mu \mathrm{g} / \mathrm{mL}$ ) complex exhibited comparable activity to that of standard drug BHT (butylated hydroxyl toluene) ( $\mathrm{IC}_{50}=$ $0.67 \mu \mathrm{g} / \mathrm{mL})$.

3.10. Cytotoxic Activity. The cytotoxicity of Schiff base ligands and their metal complexes were carried out using MTT assay. $\mathrm{IC}_{50}$ is employed to stand for the cytotoxicities of the compounds against the cancer cell lines; the smaller the $\mathrm{IC}_{50}$ value in the same condition is, the higher the cell growth inhibitory potency. DMSO when used as control without test sample, it does not exhibit any cytotoxic activity against all cancer cell lines. Cis-platin is used as positive control and it showed the highest cytotoxicity. The $\mathrm{IC}_{50}$ values were calculated after $48 \mathrm{~h}$ of incubation with compounds and are listed in Table 8. As shown in Table 8, all the ligands and their metal complexes showed lower cytotoxicity against selected cancer cell lines compared to the cis-platin. $\mathrm{HL}_{3}$ ligand showed enhanced activity against COLO 205 cell lines compared to all ligands and metal complexes $\left(\mathrm{IC}_{50}=\right.$ $15.2 \mu \mathrm{g} / \mathrm{mL}$ ). The lowest $\mathrm{IC}_{50}$ values (33.1 and $22.6 \mu \mathrm{g} / \mathrm{mL}$ ) are observed for $\mathrm{Zn}(\mathrm{II})$ complex of $\mathrm{HL}_{3}$ ligand against raw and 


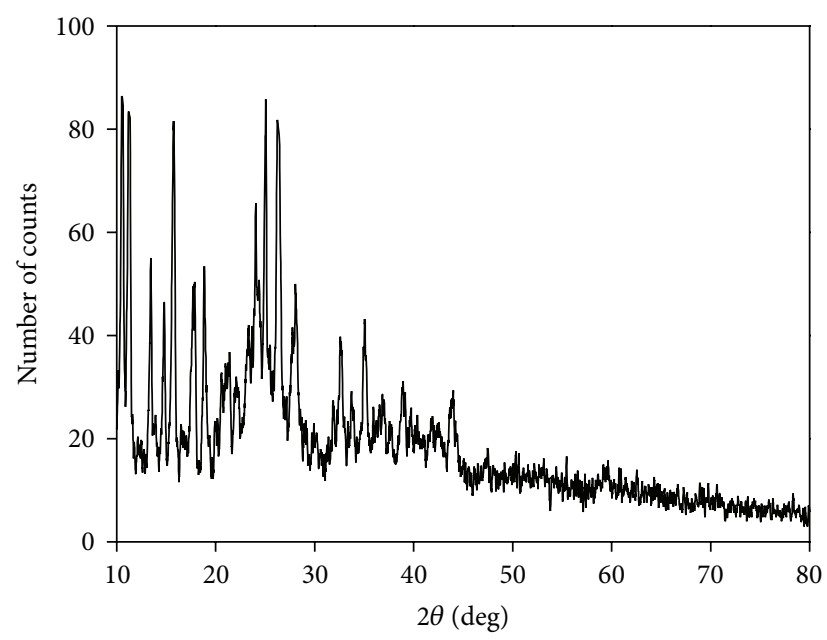

(a)

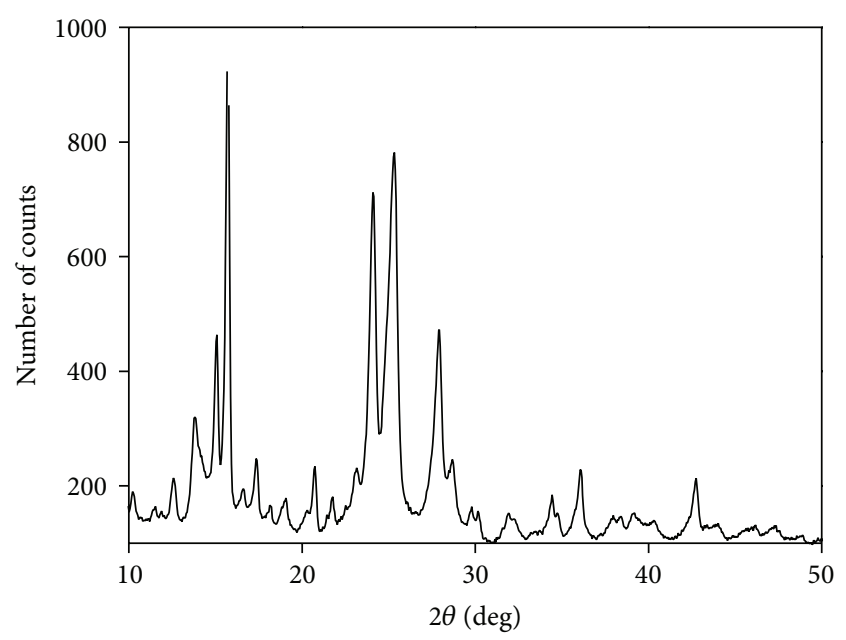

(b)

FIgure 5: Powder XRD patterns of (a) $\left[\mathrm{Ni}\left(\mathrm{L}_{1}\right)_{2}\right]$ (b) $\left[\mathrm{Zn}\left(\mathrm{L}_{1}\right)_{2}\right] \cdot \mathrm{H}_{2} \mathrm{O}$.

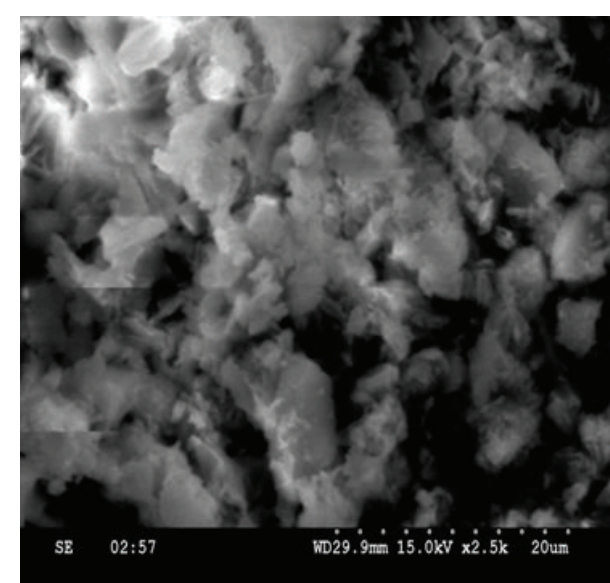

(a)

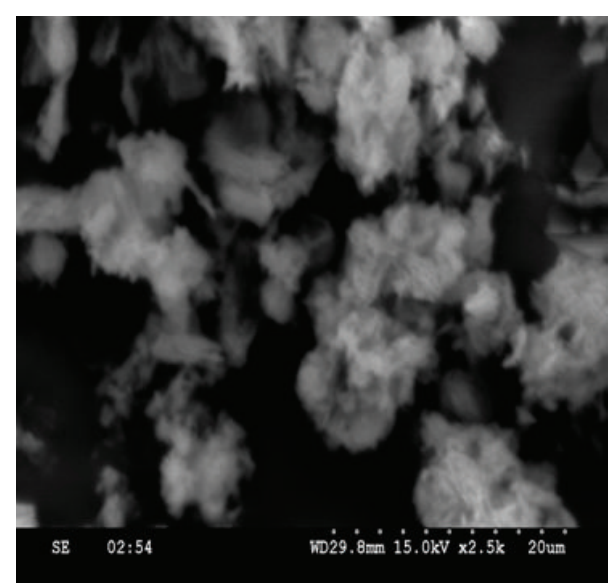

(b)

FIgURE 6: SEM micrograph of (a) $\left[\mathrm{Ni}\left(\mathrm{L}_{3}\right)_{2}\right] \cdot \mathrm{H}_{2} \mathrm{O}$ and (b) $\left[\mathrm{Ni}\left(\mathrm{L}_{4}\right)_{2}\right]$.

MCF-7 cell lines among all the ligands and metal complexes indicating its significant potency against two cancer cell lines. The results revealed that pyridine ring may be playing an important role in the cytotoxicity of the compounds.

3.11. DNA Cleavage Activity. pUC19 DNA was chosen for the DNA cleavage activity of the complexes. The studies were done using gel electrophoresis. Its naturally occurring supercoiled form (Form I) when nicked gives rise to an open circular relaxed form (Form II) and upon further cleavage results in the linear form (Form III). When subjected to gel electrophoresis, Form I migrates relatively faster while the nicked form (Form II) migrates slowly and linearized Form III migrates between Form I and Form II [45]. In the present study gel electrophoresis experiments were performed in the presence and absence of an oxidizing agent $\mathrm{H}_{2} \mathrm{O}_{2}$ to investigate the mechanism of nucleolytic activity of the complexes. The activity was greater for the complexes in the presence of $\mathrm{H}_{2} \mathrm{O}_{2}$. Control (DNA alone) does not show activity (Figure 8(a)). When $\mathrm{FeSO}_{4}$ is used as standard, it shows complete DNA cleavage. In the absence of $\mathrm{H}_{2} \mathrm{O}_{2}$ all complexes show partial nucleolytic activity. Probably this may be due to the redox behaviour of the metal ions. These results indicated the important role of the metal ions in cleavage studies. In the presence of $\mathrm{H}_{2} \mathrm{O}_{2}$, (Figure 8(b)) absence of marker bands was observed in all the complexes except $\left[\mathrm{Ni}\left(\mathrm{L}_{3}\right)_{2}\right] \cdot \mathrm{H}_{2} \mathrm{O}$ and $\left[\mathrm{Zn}\left(\mathrm{L}_{3}\right)_{2}\right] ;\left[\mathrm{Zn}\left(\mathrm{L}_{4}\right)_{2}\right] \cdot \mathrm{H}_{2} \mathrm{O}$ complexes indicate the complete DNA cleavage activity. In the case of $\left[\mathrm{Ni}\left(\mathrm{L}_{3}\right)_{2}\right] \cdot \mathrm{H}_{2} \mathrm{O},\left[\mathrm{Zn}\left(\mathrm{L}_{3}\right)_{2}\right]$, and $\left[\mathrm{Zn}\left(\mathrm{L}_{4}\right)_{2}\right] \cdot \mathrm{H}_{2} \mathrm{O}$ complexes a decrease in the intensity of bands was observed compared to the control. This is probably due to the partial cleavage of the DNA. The DNA cleavage activity of the complexes in the presence of $\mathrm{H}_{2} \mathrm{O}_{2}$ may be due to the reaction of hydroxy radicals with $\mathrm{DNA}$. The general oxidative 


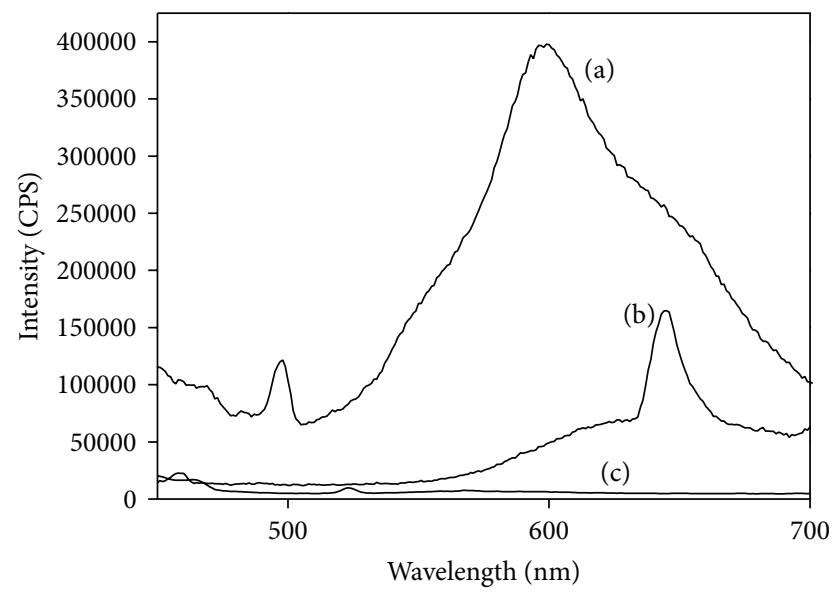

FIGURE 7: Fluorescence spectra of (a) $\mathrm{HL}_{1}$, (b) $\left[\mathrm{Ni}\left(\mathrm{L}_{1}\right)_{2}\right]$, and (c) $\left[\mathrm{Zn}\left(\mathrm{L}_{1}\right)_{2}\right] \cdot \mathrm{H}_{2} \mathrm{O}$.

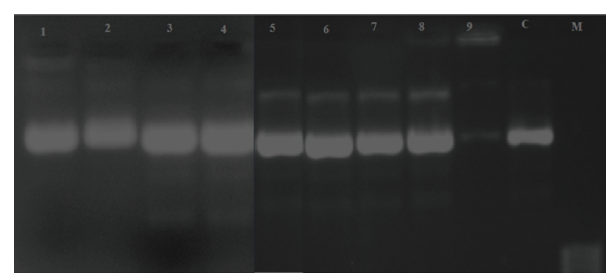

(a)

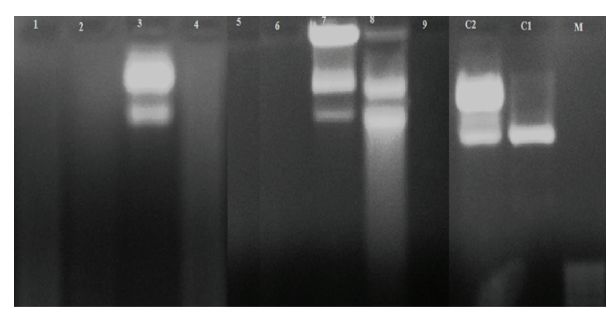

(b)

Figure 8: Gel electrophoresis photograph of metal complexes. (a) Gel electrophoresis photograph showing the effects of metal complexes on pUC 19 DNA: lane $1, \mathrm{DNA}+\left[\mathrm{Ni}\left(\mathrm{L}_{1}\right)_{2}\right]$; lane 2, DNA $+\left[\mathrm{Ni}\left(\mathrm{L}_{2}\right)_{2}\right] \cdot \mathrm{H}_{2} \mathrm{O}$; lane 3, DNA $+\left[\mathrm{Ni}\left(\mathrm{L}_{3}\right)_{2}\right] \cdot \mathrm{H}_{2} \mathrm{O}$; lane 4 , DNA + $\left[\mathrm{Ni}\left(\mathrm{L}_{4}\right)_{2}\right] ;$ lane 5, DNA + $\left[\mathrm{Zn}\left(\mathrm{L}_{1}\right)_{2}\right] \cdot \mathrm{H}_{2} \mathrm{O}$; lane 6, DNA + $\left[\mathrm{Zn}\left(\mathrm{L}_{2}\right)_{2}\right]$; lane 7, DNA + $\left[\mathrm{Zn}\left(\mathrm{L}_{3}\right)_{2}\right]$; lane 8, DNA + $\left[\mathrm{Zn}\left(\mathrm{L}_{4}\right)_{2}\right] \cdot \mathrm{H}_{2} \mathrm{O}$; lane 9, DNA $+\mathrm{FeSO}_{4}$; lane $\mathrm{C}$, DNA alone. (b) Gel electrophoresis photograph showing the effects of metal complexes on pUC 19DNA in the presence of $\mathrm{H}_{2} \mathrm{O}_{2}$ : lane $1, \mathrm{DNA}+\left[\mathrm{Ni}\left(\mathrm{L}_{1}\right)_{2}\right]+\mathrm{H}_{2} \mathrm{O}_{2}$; lane 2 , DNA + $\left[\mathrm{Ni}\left(\mathrm{L}_{2}\right)_{2}\right] \cdot \mathrm{H}_{2} \mathrm{O}+\mathrm{H}_{2} \mathrm{O}_{2}$; lane 3, DNA $+\left[\mathrm{Ni}\left(\mathrm{L}_{3}\right)_{2}\right] \cdot \mathrm{H}_{2} \mathrm{O}+\mathrm{H}_{2} \mathrm{O}_{2}$; lane 4 , DNA + $\left[\mathrm{Ni}\left(\mathrm{L}_{4}\right)_{2}\right]+\mathrm{H}_{2} \mathrm{O}_{2}$; lane 5, DNA $+\left[\mathrm{Zn}\left(\mathrm{L}_{1}\right)_{2}\right] \cdot \mathrm{H}_{2} \mathrm{O}+\mathrm{H}_{2} \mathrm{O}_{2}$; lane 6, DNA + $\left[\mathrm{Zn}\left(\mathrm{L}_{2}\right)_{2}\right]+\mathrm{H}_{2} \mathrm{O}_{2}$; lane 7, DNA + $\left[\mathrm{Zn}\left(\mathrm{L}_{3}\right)_{2}\right]+\mathrm{H}_{2} \mathrm{O}_{2}$; lane 8, DNA + $\left[\mathrm{Zn}\left(\mathrm{L}_{4}\right)_{2}\right] \cdot \mathrm{H}_{2} \mathrm{O}+\mathrm{H}_{2} \mathrm{O}_{2}$; lane 9, DNA + $\mathrm{FeSO}_{4}+\mathrm{H}_{2} \mathrm{O}_{2}$; lane $\mathrm{C} 2$, DNA $+\mathrm{H}_{2} \mathrm{O}_{2}$; lane $\mathrm{C} 1$, DNA alone.

mechanisms of the DNA cleavage studies were reported by several research groups [46-48]. Many literature reports infer that the compound was to cleave the DNA; it can be concluded that the compound inhibits the growth of the pathogenic organism by cleaving the genome [49].

\section{Conclusions}

$\mathrm{Ni}$ (II) and $\mathrm{Zn}$ (II) complexes have been synthesized using 3formyl chromone Schiff bases and characterized by various analytical and spectral data. Based on the electronic spectra, magnetic moment, and elemental analysis data, octahedral geometry was proposed for $\mathrm{Ni}$ (II) and $\mathrm{Zn}$ (II) complexes. The well-defined crystalline and homogeneous nature of the metal complexes is observed from powder XRD and SEM analyses. The antimicrobial activity data has shown that $\left[\mathrm{Zn}\left(\mathrm{L}_{3}\right)_{2}\right]$ complex displayed higher activity among all other metal complexes. The nematicidal activity of metal complexes revealed that $\left[\mathrm{Ni}\left(\mathrm{L}_{3}\right)_{2}\right] \cdot \mathrm{H}_{2} \mathrm{O}$ and $\left[\mathrm{Zn}\left(\mathrm{L}_{3}\right)_{2}\right]$ complexes showed moderate activity. $\left[\mathrm{Zn}\left(\mathrm{L}_{4}\right)_{2}\right] \cdot \mathrm{H}_{2} \mathrm{O}$ complex exhibited greater antioxidant activity compared to the remaining metal complexes. All metal complexes exhibited considerable cytotoxic activity against Raw, MCF-7, and COLO 205 cell lines. The DNA cleavage studies of metal complexes showed more prominent activity in the presence of $\mathrm{H}_{2} \mathrm{O}_{2}$ compared to that in the absence of $\mathrm{H}_{2} \mathrm{O}_{2}$.

\section{Conflict of Interests}

The authors declare that there is no conflict of interests regarding the publication of this paper.

\section{Acknowledgments}

The authors wish to thank Dr. M.R.P. Reddy, CMET (Centre for the Materials for Electronic Technologies), Hyderabad, for providing TG facility. The authors are thankful to the Department of Biochemistry, SV University, Tirupathi, for antioxidant activity study, Vimta Labs Ltd., Hyderabad, for DNA cleavage studies, and PSG college of Pharmacy, Coimbatore, Tamilnadu, for anticancer activity studies. The authors also wish to thank the Ministry of Human Resource Development for granting the research fellowship to Kavitha Palakuri.

\section{References}

[1] N. K. Singh, A. Srivastava, A. Sodhi, and P. Ranjan, "In vitro and in vivo antitumour studies of a new thiosemicarbazide derivative and its complexes with $3 \mathrm{~d}$-metal ions," Transition Metal Chemistry, vol. 25, no. 2, pp. 133-140, 2000.

[2] N. Raman, K. Pothiraj, and T. Baskaran, "DNA interaction, antimicrobial, electrochemical and spectroscopic studies of metal(II) complexes with tridentate heterocyclic Schiff base derived from $2^{\prime}$-methylacetoacetanilide," Journal of Molecular Structure, vol. 1000, no. 1-3, pp. 135-144, 2011.

[3] C. Yuan, L. Lu, Y. Wu et al., "Synthesis, characterization, and protein tyrosine phosphatases inhibition activities of oxovanadium(IV) complexes with Schiff base and polypyridyl derivatives," Journal of Inorganic Biochemistry, vol. 104, no. 9, pp. 978-986, 2010.

[4] Y. Li, Z.-Y. Yang, and J.-C. Wu, "Synthesis, crystal structures, biological activities and fluorescence studies of transition metal complexes with 3-carbaldehyde chromone thiosemicarbazone," European Journal of Medicinal Chemistry, vol. 45, no. 12, pp. 5692-5701, 2010. 
[5] Y. S. Shim, K. C. Kim, D. Y. Chi, K.-H. Lee, and H. Cho, "Formylchromone derivatives as a novel class of protein tyrosine phosphatase 1B inhibitors," Bioorganic and Medicinal Chemistry Letters, vol. 13, no. 15, pp. 2561-2563, 2003.

[6] K. M. Khan, N. Ambreen, U. R. Mughal, S. Jalil, S. Perveen, and M. I. Choudhary, "3-formylchromones: potential antiinflammatory agents," European Journal of Medicinal Chemistry, vol. 45, no. 9, pp. 4058-4064, 2010.

[7] G. Mhaske, P. Nilkanth, A. Auti, S. Davange, and S. Shelke, "Aqua medicated, microwave assisted, synthesis of Schiff bases and their biological evaluation," International Journal of Innovative Research in Science, Engineering and Technology, vol. 3, pp. 8156-8162, 2014.

[8] M. T. Hussain, N. H. Rama, and K. M. Khan, "A novel unusual isocoumarin derivative, $3 \mathrm{H}$-Furo $[3,4-c]$ isochromene1, 5-dione," Letters in Organic Chemistry, vol. 7, no. 7, pp. 557$560,2010$.

[9] A. A. Osowole, "Synthesis, characterization, in-vitro antibacterial and anticancer studies on some metal(II) complexes of (methylsulfanyl)chromenol Schiff base," Elixir Applied Chemistry, vol. 39, pp. 4827-4831, 2011.

[10] K. M. Khan, A. Ahmad, N. Ambreen et al., "Schiff bases of 3formylchromones as antibacterial, antifungal, and phytotoxic agents," Letters in Drug Design and Discovery, vol. 6, no. 5, pp. 363-373, 2009.

[11] S. Bhatnagar, S. Sahi, P. Kackar et al., "Synthesis and docking studies on styryl chromones exhibiting cytotoxicity in human breast cancer cell line," Bioorganic and Medicinal Chemistry Letters, vol. 20, no. 16, pp. 4945-4950, 2010.

[12] A. Gomes, O. Neuwirth, M. Freitas et al., "Synthesis and antioxidant properties of new chromone derivatives," Bioorganic \& Medicinal Chemistry, vol. 17, no. 20, pp. 7218-7226, 2009.

[13] L. Z. Piao, H. R. Park, Y. K. Park, S. K. Lee, J. H. Park, and M. K. Park, "Mushroom tyrosinase inhibition activity of some chromones," Chemical and Pharmaceutical Bulletin, vol. 50, no. 3, pp. 309-311, 2002.

[14] S. A. Patil, S. N. Unki, A. D. Kulkarni, V. H. Naik, and P. S. Badami, "Synthesis, characterization, in vitro antimicrobial and DNA cleavage studies of $\mathrm{Co}(\mathrm{II}), \mathrm{Ni}(\mathrm{II})$ and $\mathrm{Cu}(\mathrm{II})$ complexes with ONOO donor coumarin Schiff bases," Journal of Molecular Structure, vol. 985, no. 2-3, pp. 330-338, 2011.

[15] F. Arjmand, F. Sayeed, and M. Muddassir, "Synthesis of new chiral heterocyclic Schiff base modulated $\mathrm{Cu}(\mathrm{II}) / \mathrm{Zn}$ (II) complexes: their comparative binding studies with CT-DNA, mononucleotides and cleavage activity," Journal of Photochemistry and Photobiology B: Biology, vol. 103, no. 2, pp. 166-179, 2011.

[16] C. N. Sudhamani, H. S. B. Naik, T. R. R. Naik, and M. C. Prabhakara, "Synthesis, DNA binding and cleavage studies of $\mathrm{Ni}(\mathrm{II})$ complexes with fused aromatic $\mathrm{N}$-containing ligands," Spectrochimica Acta A: Molecular and Biomolecular Spectroscopy, vol. 72, no. 3, pp. 643-647, 2009.

[17] D.-D. Qin, Z.-Y. Yang, and B.-D. Wang, "Spectra and DNAbinding affinities of copper(II), nickel(II) complexes with a novel glycine Schiff base derived from chromone," Spectrochimica Acta A: Molecular and Biomolecular Spectroscopy, vol. 68, no. 3, pp. 912-917, 2007.

[18] B.-D. Wang, Z.-Y. Yang, M.-H. Lü, J. Hai, Q. Wang, and Z.N. Chen, "Synthesis, characterization, cytotoxic activity and DNA binding $\mathrm{Ni}(\mathrm{II})$ complex with the 6-hydroxy chromone3-carbaldehyde thiosemicarbazone," Journal of Organometallic Chemistry, vol. 694, no. 25, pp. 4069-4075, 2009.
[19] Y. Li, Z. Y. Yang, Z. C. Liao, Z. C. Han, and Z. C. Liu, "Synthesis, crystal structure, DNA binding properties and antioxidant activities of transition metal complexes with 3carbaldehyde-chromone semicarbazone," Inorganic Chemistry Communications, vol. 13, no. 10, pp. 1213-1216, 2010.

[20] B.-D. Wang, Z.-Y. Yang, and T.-R. Li, "Synthesis, characterization, and DNA-binding properties of the $\operatorname{Ln}(\mathrm{III})$ complexes with 6-hydroxy chromone-3-carbaldehyde-(2'-hydroxy) benzoyl hydrazone," Bioorganic and Medicinal Chemistry, vol. 14, no. 17, pp. 6012-6021, 2006.

[21] P. Kavitha, M. Saritha, and K. L. Reddy, "Synthesis, structural characterization, fluorescence, antimicrobial, antioxidant and DNA cleavage studies of $\mathrm{Cu}$ (II) complexes of formyl chromone Schiff bases," Spectrochimica Acta A: Molecular and Biomolecular Spectroscopy, vol. 102, pp. 159-168, 2013.

[22] P. Kavitha, M. R. Chary, B. V. A. A. Singavarapu, and K. L. Reddy, "Synthesis, characterization, biological activity and DNA cleavage studies of tridentate Schiff bases and their Co(II) complexes," Journal of Saudi Chemical Society, 2013.

[23] P. Kavitha and K. L. Reddy, "Pd(II) complexes bearing chromone based Schiff bases: synthesis, characterisation and biological activity studies," Arabian Journal of Chemistry, 2012.

[24] A. Nohara, T. Umetani, and Y. Sanno, "A facile synthesis of chromone-3-carboxaldehyde, chromone-3-carboxylic acid and 3-hydroxymethylchromone," Tetrahedron Letters, vol. 14, no. 22, pp. 1995-1998, 1973.

[25] I. Sigg, G. Haas, and T. Winkler, "The reaction of 3formylchromone with ortho-substituted anilines. Preparation of a Tetraaza [14] annulene," Helevetica Chimica Acta, vol. 65, no. 1, pp. 275-279, 1982.

[26] K. M. Khan, N. Ambreen, S. Hussain, S. Perveen, and M. I. Choudhary, "Schiff bases of 3-formylchromone as thymidine phosphorylase inhibitors," Bioorganic and Medicinal Chemistry, vol. 17, no. 8, pp. 2983-2988, 2009.

[27] J. E. Stemper and J. M. Matsen, "Device for turbidity standardizing of cultures for antibiotic sensitivity testing," Applied Microbiology, vol. 19, no. 6, pp. 1015-1016, 1970.

[28] K. Shanker, R. Rohini, V. Ravinder, P. M. Reddy, and Y.-P. Ho, "Ru(II) complexes of $\mathrm{N}_{4}$ and $\mathrm{N}_{2} \mathrm{O}_{2}$ macrocyclic Schiff base ligands: their antibacterial and antifungal studies," Spectrochimica Acta A: Molecular and Biomolecular Spectroscopy, vol. 73, no. 1, pp. 205-211, 2009.

[29] P. C. Trivedi, Nematode Diseases in Plants, CBS, New Delhi, India, 1st edition, 1998.

[30] J. Cayrol, C. Djian, and L. Pijarowski, "Study of the nematicidal properties of the culture filtrate of the nematophagous fungus Paecilomyces lilacinus," Revue de Nematologie, vol. 12, pp. 331336, 1989.

[31] A. Braca, N. de Tommasi, L. di Bari, C. Pizza, M. Politi, and I. Morelli, "Antioxidant principles from Bauhinia tarapotensis," Journal of Natural Products, vol. 64, no. 7, pp. 892-895, 2001.

[32] S. Sathiyaraj, R. J. Butcher, and Ch. Jayabalakrishnan, "Synthesis, characterization, DNA interaction and in vitro cytotoxicity activities of ruthenium(II) Schiff base complexes," Journal of Molecular Structure, vol. 1030, pp. 95-103, 2012.

[33] W. J. Geary, "The use of conductivity measurements in organic solvents for the characterisation of coordination compounds," Coordination Chemistry Reviews, vol. 7, no. 1, pp. 81-122, 1971.

[34] A. I. Vogel, A Text Book of Quantitative Inorganic Analysis, Longman, 3rd edition, 1961. 
[35] O. A. El-Gammal, G. A. El-Reash, and S. F. Ahmed, "Structural, spectral, thermal and biological studies on 2 -oxo- $\mathrm{N}^{\prime}-((4-$ oxo-4H-chromen-3-yl)methylene)-2-(phenylamino)acetohydrazide $\left(\mathrm{H}_{2} \mathrm{~L}\right)$ and its metal complexes," Journal of Molecular Structure, vol. 1007, pp. 1-10, 2012.

[36] C. Anitha, C. D. Sheela, P. Tharmaraj, and S. J. Raja, "Synthesis and characterization of $\mathrm{VO}(\mathrm{II}), \mathrm{Co}(\mathrm{II}), \mathrm{Ni}(\mathrm{II}), \mathrm{Cu}(\mathrm{II})$ and $\mathrm{Zn}$ (II) complexes of chromone based azo-linked Schiff base ligand," Spectrochimica Acta A: Molecular and Biomolecular Spectroscopy, vol. 98, pp. 35-42, 2012.

[37] M. Padmaja, J. Pragathi, and C. G. Kumari, "Synthesis, spectral characterization, molecular modeling and biological activity of first row transition metal complexes with Schiff base ligand derived from chromone-3-carbaldehyde and o-amino benzoic acid," Journal of Chemical and Pharmaceutical Research, vol. 3, no. 4, pp. 602-613, 2011.

[38] B. Kannamba, K. L. Reddy, and B. V. AppaRao, "Removal of $\mathrm{Cu}(\mathrm{II})$ from aqueous solutions using chemically modified chitosan," Journal of Hazardous Materials, vol. 175, no. 1-3, pp. 939-948, 2010.

[39] S. Chandra and L. K. Gupta, "Spectroscopic and biological studies on newly synthesized nickel(II) complexes of semicarbazones and thiosemicarbazones," Spectrochimica Acta A: Molecular and Biomolecular Spectroscopy, vol. 62, no. 4-5, pp. 1089-1094, 2005.

[40] P. K. Singh and D. N. Kumar, "Spectral studies on cobalt(II), nickel(II) and copper(II) complexes of naphthaldehyde substituted aroylhydrazones," Spectrochimica Acta A: Molecular and Biomolecular Spectroscopy, vol. 64, no. 4, pp. 853-858, 2006.

[41] B. E. Warren, X-Ray Diffraction, Dover, New York, NY, USA, 2nd edition, 1990.

[42] X. Huang, Y. Xia, H. Zhang et al., "Synthesis, crystal structure, and fluorescence studies of (1-naphthyl)(pyridyl)urea metal complexes," Inorganic Chemistry Communications, vol. 11, no. 4, pp. 450-453, 2008.

[43] A. K. Sharma and S. Chandra, "Complexation of nitrogen and sulphur donor Schiff's base ligand to $\mathrm{Cr}$ (III) and $\mathrm{Ni}$ (II) metal ions: synthesis, spectroscopic and antipathogenic studies," Spectrochimica Acta A: Molecular and Biomolecular Spectroscopy, vol. 78, no. 1, pp. 337-342, 2011.

[44] T. A. Yousef, G. M. Abu El-Reash, O. A. El-Gammal, and R. A. Bedier, "Co(II), $\mathrm{Cu}(\mathrm{II}), \mathrm{Cd}(\mathrm{II}), \mathrm{Fe}(\mathrm{III})$ and U(VI) complexes containing a NSNO donor ligand: synthesis, characterization, optical band gap, in vitro antimicrobial and DNA cleavage studies," Journal of Molecular Structure, vol. 1029, pp. 149-160, 2012.

[45] H.-L. Seng, H.-K. A. Ong, R. N. Z. R. A. Rahman et al., "Factors affecting nucleolytic efficiency of some ternary metal complexes with DNA binding and recognition domains. Crystal and molecular structure of $\mathrm{Zn}$ (phen)(edda)," Journal of Inorganic Biochemistry, vol. 102, no. 11, pp. 1997-2011, 2008.

[46] S. K. Bharti, S. K. Patel, G. Nath, R. Tilak, and S. K. Singh, "Synthesis, characterization, DNA cleavage and in vitro antimicrobial activities of copper(II) complexes of Schiff bases containing a 2,4-disubstituted thiazole," Transition Metal Chemistry, vol. 35, no. 8, pp. 917-925, 2010.

[47] D. S. Sigman, A. Mazumder, and D. M. Perrin, "Chemical nucleases," Chemical Reviews, vol. 93, no. 6, pp. 2295-2316, 1993.

[48] T. Kobayashi, M. Kunita, S. Nishino et al., "Release of free nucleobases from oligomers by copper(II)-peroxide adduct," Polyhedron, vol. 19, no. 26-27, pp. 2639-2648, 2000.
[49] T. A. Yousef, G. M. Abu El-Reash, O. A. El-Gammal, and R. A. Bedier, "Synthesis, characterization, optical band gap, in vitro antimicrobial activity and DNA cleavage studies of some metal complexes of pyridyl thiosemicarbazone," Journal of Molecular Structure, vol. 1035, pp. 307-317, 2013. 

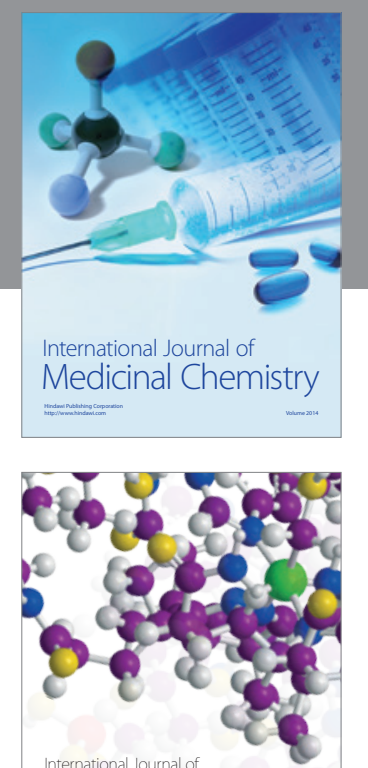

\section{Carbohydrate} Chemistry

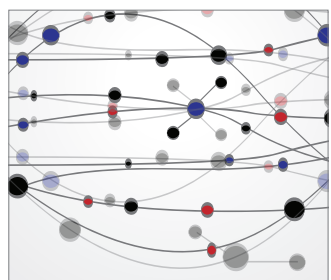

The Scientific World Journal
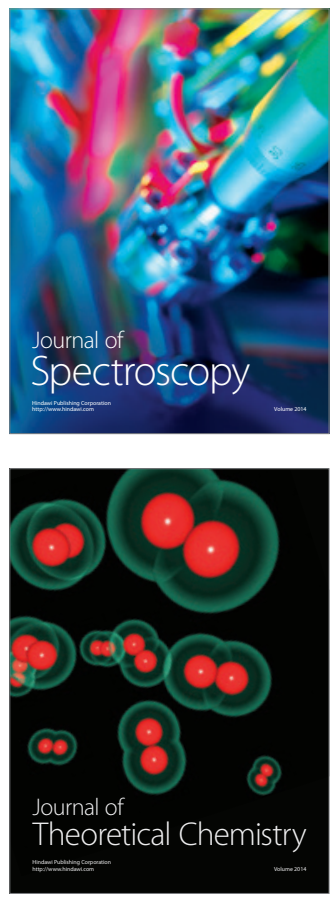
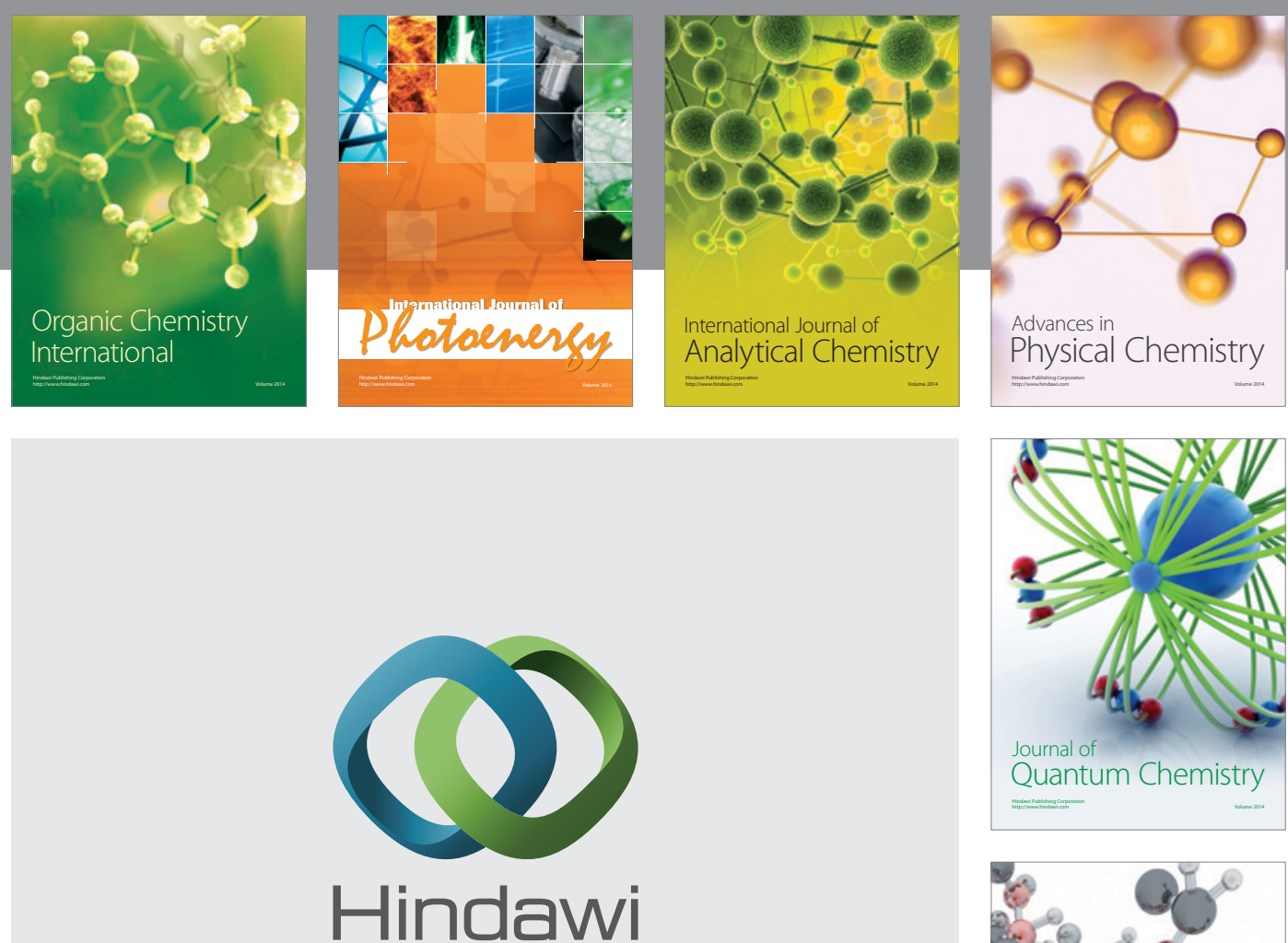

Submit your manuscripts at

http://www.hindawi.com

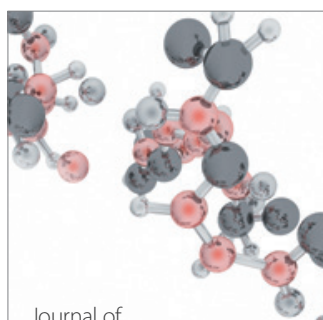

Analytical Methods

in Chemistry

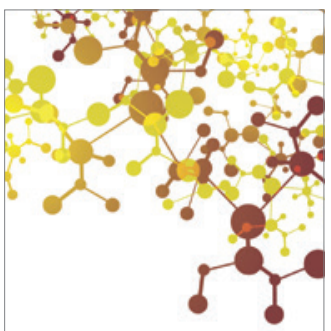

Journal of

Applied Chemistry

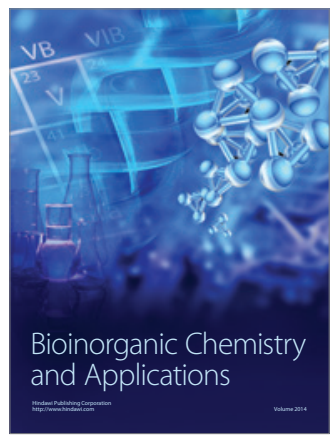

Inorganic Chemistry
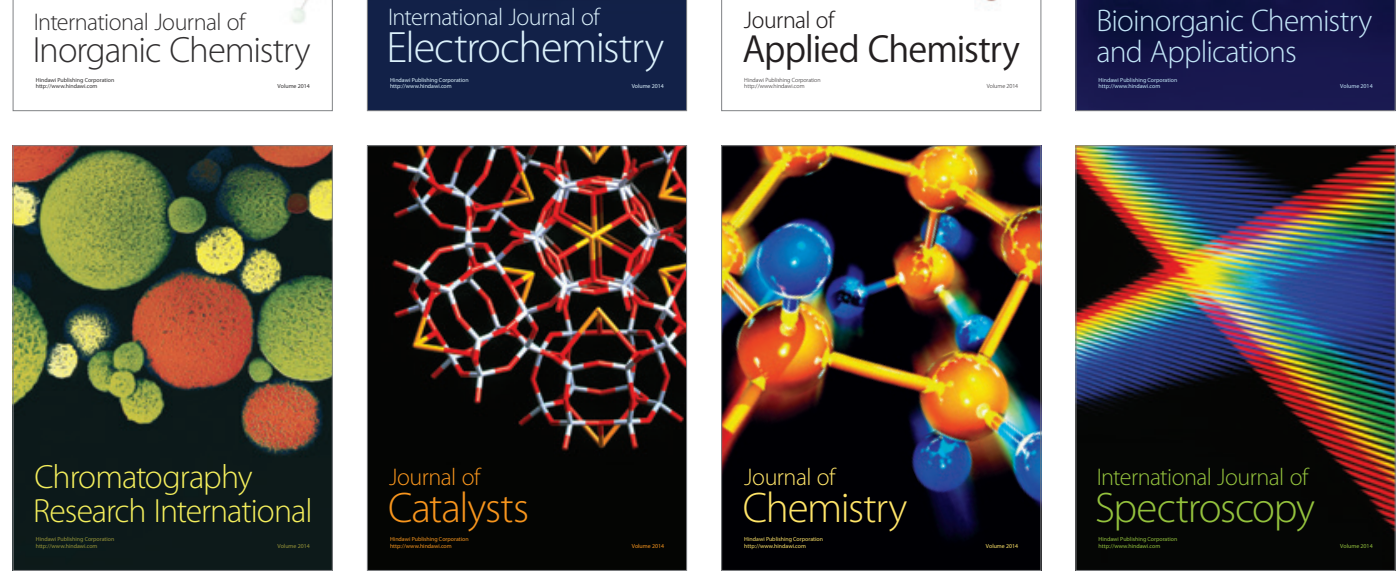